\title{
ABSTRACT \\ REGIOSELECTIVITY IN FREE RADICAL BROMINATION OF UNSYMMETRICAL DIMETHYLATED PYRIDINES
}

\author{
by Rajesh Thapa
}

During a literature review some curious inconsistencies in the free radical bromination of picolines were noted. To achieve a better understanding of the mechanisms and regioselectivity we reran these reactions, extending our work to unsymmetrical lutidines using N-bromosuccinimide in limiting amount. Characterization of the products was done with GC/MS and H NMR. The regioselectivity of bromination in unsymmetrical dimethylpyridines shows that nitrogen in the ring is deactivating inductively. The competition between 2,3, 2,4 and 2,5 dimethyl pyridine towards bromination results with bromination in the methyl group farthest from the $\mathrm{N}$ in the ring. 3,4-Lutidine shows only the 4,4- dibrominated product. 


\title{
REGIOSELECTIVITY IN FREE RADICAL BROMINATION OF UNSYMMETRICAL DIMETHYLATED PYRIDINES
}

\author{
A Thesis \\ Submitted to the \\ Faculty of Miami University \\ in partial fulfillment of \\ the requirements for the degree of \\ Master of Science \\ Department of Chemistry and Biochemistry \\ by \\ Rajesh Thapa \\ Miami University \\ Oxford, Ohio \\ 2009 \\ Chair \\ Dr. Gilbert Ellery Pacey \\ Advisor \\ Dr. Richard T. Taylor \\ Reader \\ Dr. Benjamin W. Gung \\ Reader \\ Dr. Hong Wang
}




\section{TABLE OF CONTENTS}

Page

\section{Chapter 1: Review of NBS Bromination}

1. Introduction 1

1.1: NBS Bromination 1

1.2: Bromination of aliphatic and alicyclic olefins 2

1.3: Allylic or Benzylic Bromination 3

1.4: Unsaturation and Aromatization Reactions $\quad 7$

1.5: $\alpha$-Bromination of Carbonyl Derivatives $\quad 8$

1.6: Reaction with Vinylic and Alkynic Derivatives 9

1.7: Free Radical Bromination of Oligopyridine Benzylic-methyl Group 13

1.8: Solvents: NBS Bromination 14

1.9: NBS under Solvent Free Conditions 18

1.10: Regioselective bromination of Polymethylpyrimidines 19

1.11: An Unusual Orienting effect in the Bromination of Alkyl Bromides 23

1.12: Electronic effect in selectivity $\sigma^{+}$value for different position of pyridine $\quad 26$

1.13: Free Radical Halogenation $\quad 27$

1.14: Chorination with $N$-Chlorosuccinimide 29

1.15: Selectivity of free-radical halogenations 30

1.16: Hammond's Postulates 32

1.17: Reported reactions of picoline 35

1.18: Reported reactions of lutidine 36

1.19: Research Aim 38 
Chapter 2: Experiment, Results and Discussion

2.1 General Reaction Protocol 39

2.2 GC/MS analysis $\quad 39$

2.3 Standard Procedure $\quad 39$

2.3.1 .Bromination of 2-Methylpyridine $\quad 40$

2.3.2 Bromination of 3-Methylpyridine 40

2.3.3 Bromination of 4-Methylpyridine 41

2.3.4 Bromination of 2, 4-dimethylpyridine 42

2.3.5 Bromination of 2, 5-dimethylpyridine 42

2.3.6 Bromination of 3, 4-dimethylpyridine 43

2.3.7 Bromination of 2, 3-dimethylpyridine 44

3. Results and Discussion 44

4. Conclusion 46

5. Future Work 46

References 


\section{LIST OF TABLES}

\section{Table}

\section{Page}

Table 1: Preparation of Benzylic Bromides

Table 2: Bromination of (30) with NBS

Table 3: Benzylic bromination by NBS in (trifluoromethyl) benzene 17

Table 4: Bond Dissociation Enthalpies for hydrocarbons

Table 5: Reactants and products of NBS bromination

Table 6: Reactants and products of NBS bromination 


\section{LIST OF SCHEMES}

$\begin{array}{lll}\text { Scheme } & \text { Page }\end{array}$

Scheme 1: Free radical bromination of gem-dihaloalkenes 4

Scheme 2: Nuclear Bromination mechanism by NBS 11

Scheme 3: Free Radical Bromination mechanism by NBS 12

Scheme 4: NBS Bromination of Toluene and substituted Toluene 14

Scheme 5: Free Radical Bromination of Benzylic Methyl group 18

Scheme 6: Selective Bromination with $\mathrm{Br}_{2} / \mathrm{AcOH} \quad 19$

Scheme 7: Bromination of Dimethyl pyridines $\quad 21$

$\begin{array}{lr}\text { Scheme 8: Bromination of compound (48) } & 21\end{array}$

Scheme 9: Bromination of Non-hindered Methyl Pyridine 22 


\section{LIST OF ABBREVIATIONS}

\section{Abbreviations}

TLC

HNMR

NOE

NOSEY

HPLC

GC

GC-MS

IR

v.p.c.

NBS

NCS

BPO

AIBN

ACCN

EtOAc

$\mathrm{CDCl}_{3}$

$\mathrm{MgSO}_{4}$

\section{Technique}

thin layer chromatography

proton nuclear magnetic resonance

nuclear Overhauser effect

nuclear Overhauser effect spectroscopy

high performance liquid chromatography

gas chromatography

gas chromatography-mass spectroscopy

infrared spectroscopy

Vapor pressure chromatography

\section{Chemical}

$\mathrm{N}$-bromosuccinimide

N-chlorosuccinimide

benzoyl peroxide

2, 2'-azobisisobutyronitrile

1,1'-azobis(cyclohexanecarbonitrile

ethyl acetate

deuterated chloroform

magnesium sulfate

vi 


\begin{tabular}{|c|c|}
\hline $\mathrm{MeOH}$ & methanol \\
\hline $\mathrm{CCl}_{4}$ & carbon tetrachloride \\
\hline $\mathrm{C}_{2} \mathrm{Cl}_{4}$ & tetrachloroethane \\
\hline $\mathrm{CH}_{2} \mathrm{Cl}_{2}$ & dichloroethane \\
\hline $\mathrm{AcOH}$ & acetic acid \\
\hline DDQ & 2,3-dichloro-5,6-dicyanobenzoquinone \\
\hline DMSO & dimethylsulfoxide \\
\hline DMF & dimethylformamide \\
\hline TEMPO & 2,2,6,6-tetramethylpiperdin-1-yloxy \\
\hline \multirow[t]{2}{*}{$\mathrm{LiAlH}_{4}$} & lithium Aluminum Hydride \\
\hline & Other \\
\hline EWG & electron withdrawing group \\
\hline EDG & electron donating group \\
\hline ERG & electron releasing group \\
\hline SFRC & solvent free reaction condition \\
\hline FW & formula weight \\
\hline mmol & milli mole \\
\hline ppm & parts per million \\
\hline $\mathrm{R}_{\mathrm{f}}$ & Retention factor \\
\hline $\mathrm{rt}$ & Room temperature \\
\hline m.p. & melting point \\
\hline
\end{tabular}




\section{ACKNOWLEDGEMENT}

I am grateful to my respectable advisor Dr. Richard T. Taylor, for making my time at Miami University enjoyable, productive and beneficial. I also thank him for his guidance, innovative ideas and generous support and encouragement through out this project.

Special thanks are given to my committee members Dr. Gilbert Ellery Pacey, Dr. Benjamin W. Gung, Dr. Hong Wang for their time and valuable comments, and the faculty of Department of Chemistry for coursework.

I would like to thank my Parents Shyam Dip Thapa and Kamala Thapa as well as my beloved wife Bamita Thapa, my daughter Ashmita Thapa and son Utsav Thapa for their company and constant encouragement.

Last but not the least, I would like to thank all my friends Daka, Rohit, Rip, Binay Aryal, Harish Chandra Ghimire, Yamnath Timsina and Dipendra Gautam here and back home in Nepal for their suggestions and encouragement. Special thanks to my colleague Beth (Elizabeth Goei) for her valuable comments and suggestions during my thesis writing work.

Finally, I would also like to acknowledge Miami University, Oxford, Ohio for giving me this opportunity. 


\section{CHAPTER 1: Brief Review of NBS Bromination}

\section{Introduction:}

\subsection{NBS Bromination}

Bromination is a very important process in organic synthesis as bromo derivatives serve as useful intermediates in the manufacture of pharmaceuticals, agrochemical and other specialist chemicals. ${ }^{1,2}$ Moreover, many pesticides, insecticides, herbicides and fire retardants contain the bromine functionality. ${ }^{3,4} \mathrm{~N}$-Bromosuccinimide is one of the most important brominating agents, especially for free radical bromination.

While $N$-Bromosuccinimide (NBS) is a convenient source of bromine for both substitution and electrophilic addition reactions. NBS has been extensively used for allylic and benzylic bromination. NBS has several advantages over the use of molecular bromine for these types of free radical substitution.

Mechanistic investigations have established that molecular bromine is the active halogenating agent under the usual condition used for NBS bromination. Molecular bromine is maintained at a low concentration throughout the course of reaction by formation from NBS and hydrogen bromide.

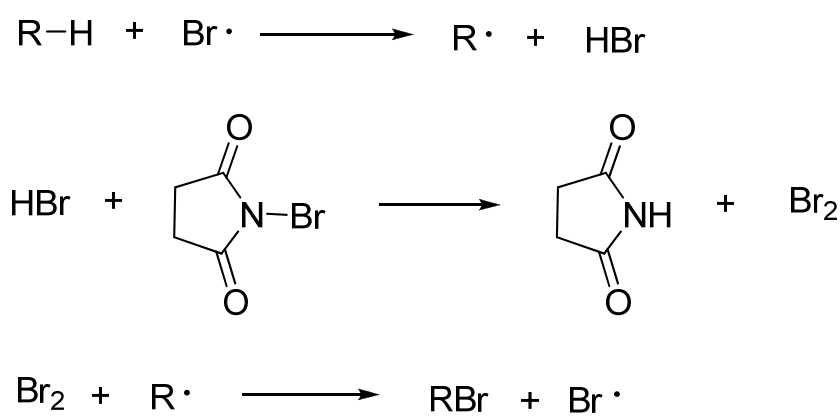

The fact that the bromine concentration remains at very low levels is important to the success of the allylic halogenation process. Substitution at allylic position in preference to addition is the result of the reversibility of electrophilic attack by bromine at the double bond. With a low concentration of bromine, the rate of bromine addition is slow enough to permit 
hydrogen atom abstraction to compete successfully. ${ }^{5} \mathrm{~N}$-Chlorosuccinimide and $\mathrm{N}$-Iodosuccinimide generally do not facilitate the analogous substitution reactions. In free radical bromination reactions with NBS, it is thought that bromine is the actual halogenating agent. The bromine generated by reaction of $\mathrm{HBr}$ obtained as the by-product of halogenation reaction with NBS.

N-bromosuccinimide can also be used to brominate alkanes. For examples, cyclopropane, cyclopentane, and cyclohexane give the corresponding bromides when irradiated with NBS in dichloromethane. ${ }^{6}$ Under these conditions, the succinimidyl radical appears to be involved as the hydrogen-abstracting intermediate.

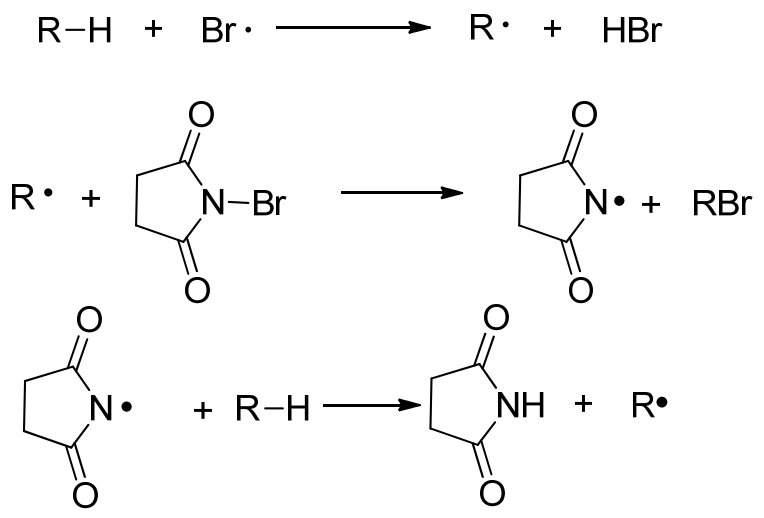

\subsection{Bromination of aliphatic and alicyclic olefins}

Allylic bromides are especially useful as either precursors for the preparation of organometallic reagents or electropiles. Among so many brominating reagents, NBS is a versatile reagent for radical bromination at the allylic position of alkenes, known as WohlZiegler bromination reaction. ${ }^{7}$ By this method, many kinds of alkenes are converted to allylic halides in reasonable (> 60\%) yields. ${ }^{8}$

The brominations of simple olefins, such as 2, 3-dimethyl-2-butene with NBS in $\mathrm{CCl}_{4}$ have been studied. ${ }^{9}$ With few exceptions NBS reacted much more readily with a methylene than a methyl group and tertiary hydrogen atoms were not attacked. 2-methyl-2-butene (1) required 16 hours and 2-methyl-hexene required 10 minutes for the completion of the reaction. A similar relationship was reported with diphenylolefins (3) and (4). 
<smiles>CC=C(C)C</smiles>

(1)

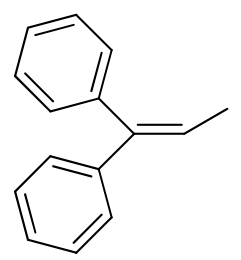

(3)

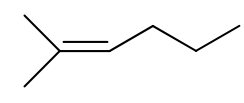

(2)

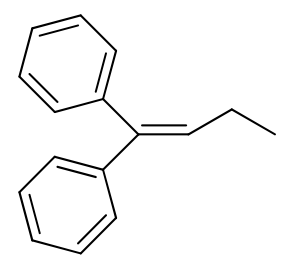

(4)

Bromination of cyclohexene (5) with NBS gave 3-bromo derivative (6) with high yield and dehydrobromination with quinoline gave 1, 3-cyclohexadiene (7) with small amount of (8) was reported.

Addition of small amount of initiators such as dibenzoyl peroxide was found to promote the free radical bromination reaction in terms of both reaction rate and yield. There is also evidence that reaction leading to the formation of $N$-phenylsuccinimide is favored by dibenzoyl peroxide. Howton ${ }^{10}$ made a detailed study of this reaction in the presence of peroxide. ${ }^{11}$

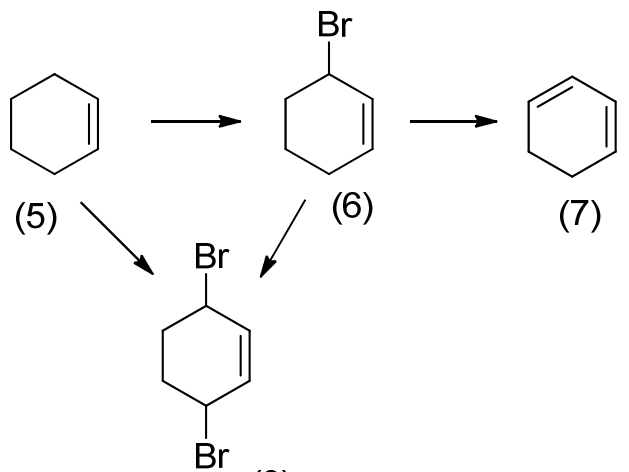

(8)

\section{3: Allylic or Benzylic Bromination}

Free radical halogenation is most commonly applied to allylic or benzylic halogenation, as the radicals formed at these positions are lowest in energy. Allylic bromination ${ }^{9}$ can be 
obtained by refluxing the alkene solution and NBS in anhydrous $\mathrm{CCl}_{4}$ using free radical initiator azo-bis-isobutyronitrile (AIBN) or benzoyl peroxide (BPO). Irradiation with visible light alternatively promotes the reaction.

High levels of ragioselectivity function during the hydrogen-abstraction steps of the chain mechanism, such that allylic methylene groups are attacked much more rapidly than allylic methyl groups. ${ }^{12}$ In other words, allylic radical intermediates formed during reaction are much more stable than other carbon radicals and the major products are allylic bromides. ${ }^{9}$ Free radical bromination of gem-dihaloalkenes (9) was reported. ${ }^{13}$ The reaction of (9) with 1.95 equivalents of NBS in the presence of $10 \%$ of BPO yield (b) with $86 \%$ yield was reported.

Scheme 1: Free Radical Bromination of gem-dihaloalkenes

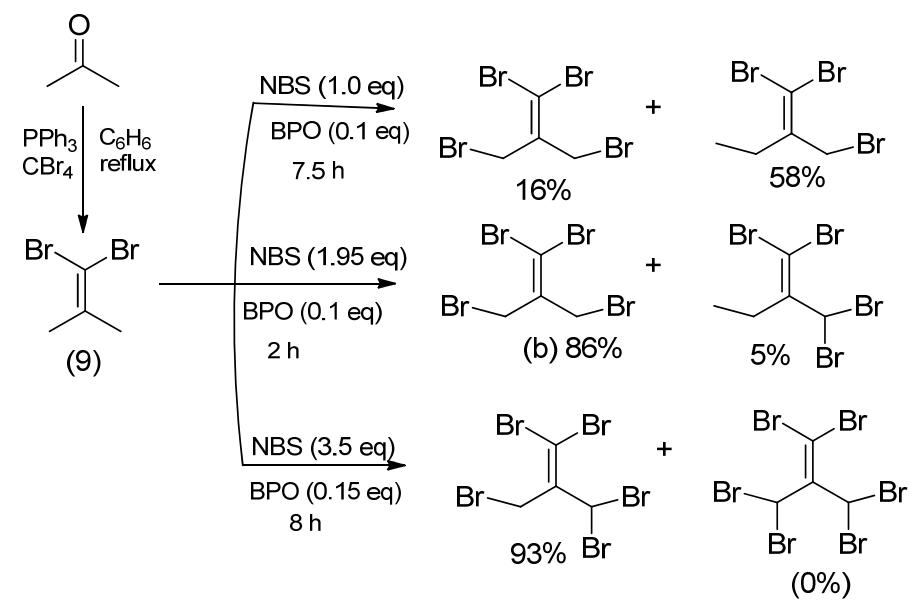

With 3.5 equivalents of NBS, (9) provided a tribrominated product in 93\% yield. Interesting results was reported that tetrabrominated products were not obtained even when 6 equivalents of NBS were used.

High levels of functional group selectivity are characteristic of radical bromination as well. For example bromination of $\alpha, \beta$-unsaturated esters (eqn. 10) ${ }^{14,15}$ and lactones (eqn. 11$)^{16}$ are reported to be very successful. 

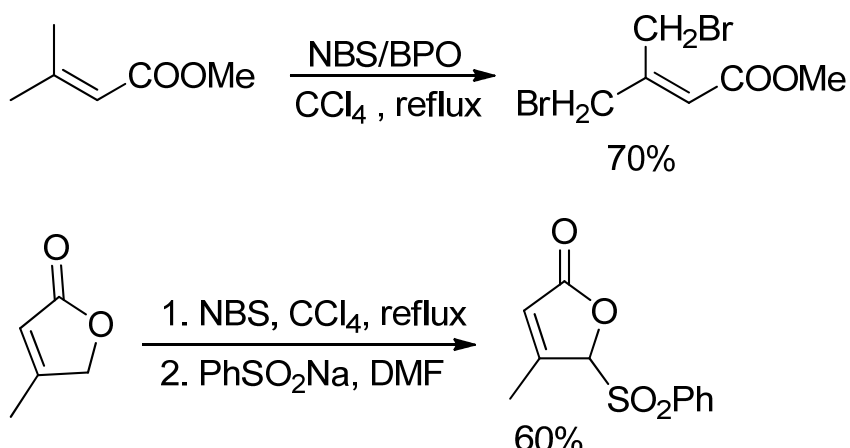

When the same conditions as allylic bromination are applied to the benzylic bromination of aromatic compounds, NBS effects the bromination of benzylic positions. ${ }^{17}$ Bromine can also be used for benzylic bromination (eqn. 12) ${ }^{16,17}$ but many functional groups are sensitive to the generation of $\mathrm{HBr}$ during the reaction which competes with acid catalyzed bromination. This makes NBS the reagent of choice for bromination of polyfunctional aromatic compounds.

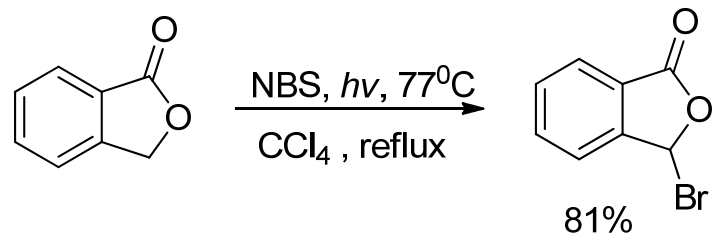

Selectivity can be predicted with polyfunctional molecules based on the stabilities of the free radical intermediates (eqn. 13). ${ }^{18}$ Accordingly, the use of NBS allows the bromination of alkyl groups attached to sensitive heterocyclic compounds (eqn. 14). ${ }^{19}$

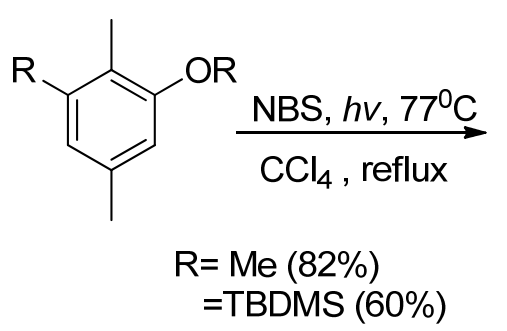<smiles>[R]c1cc(C)cc([R])c1CBr</smiles> 

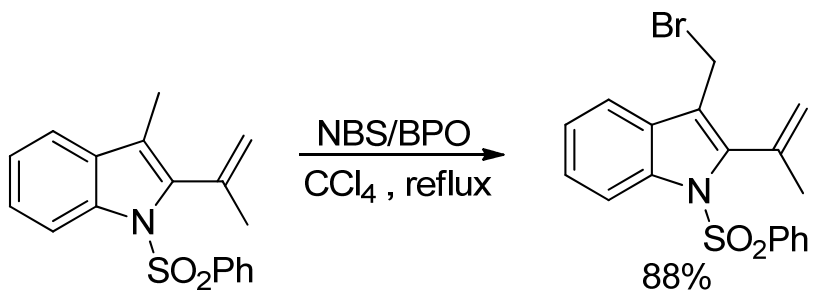

The benzylic bromides, especially the poly(monobromomethyl) are useful intermediates in organic synthesis. They are widely used in the synthesis of dendrimers, ${ }^{20}$ caged compounds, ${ }^{21}$ etc. The preparations of such compound are often carried out by converting benzylic alcohols to benzylic bromides. However, alcohols are expensive or difficult to prepare following cumbersome route. ${ }^{22,23}$ Benzylic bromination with NBS is an important alternative. The free radical bromination gives a $67 \%$ yield of benzyl bromide from toluene. This is not satisfactory because of the following three reasons. (i). Multiple brominations cause the yield to be low. (ii). The byproducts, polybromides and monobromides have the same $\mathrm{R}_{\mathrm{f}}$ value in TLC, which make purification difficult. (iii). Electron withdrawing substituent and heteroatom make the yield low.eg, 2,6-lutidine give only $2 \%$ bis(bromomethyl)pyridine in $\mathrm{CCl}_{4}{ }^{24,25}$

An efficient method for the preparation of benzylic bromides is reported. ${ }^{26}$ Substituted benzylic compounds are brominated with excess of $\mathrm{NBS}$ in $\mathrm{CCl}_{4}$ to the corresponding polybrominated mixtures which are then debrominated with diethyl phosphate and $N, N$ diisopropylethylamine to give the desired monobromides in satisfactory yields and high purity. The scheme is as follows.

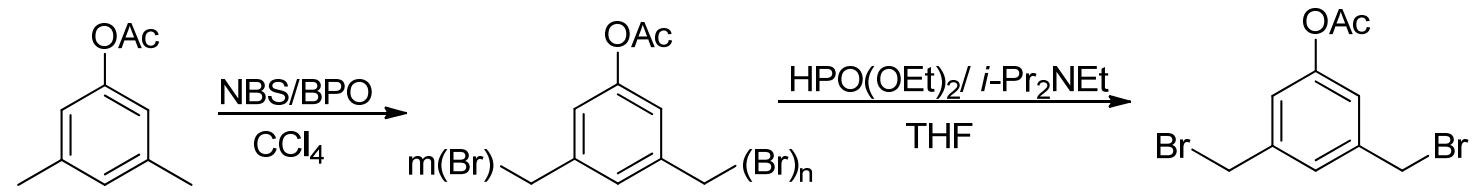
$\mathrm{m}, \mathrm{n}=1,2,3$

This convenient two step procedure for the preparation of benzylic bromides from methyl benzenes has been generalized. The benzylic methylcompounds are first brominated with an excess of NBS in $\mathrm{CCl}_{4}$ and then selectively debrominated to the monobromides with diethyl 
phosphate and $N, N$-diisopropylethylamine in satisfactory yields. High purity was reported. The synthesis of benzyl bromides reported is as follows. ${ }^{26}$

Table 1: Preparation of Benzylic Bromides

\begin{tabular}{|l|l|l|l|}
\hline Substrate & \multicolumn{2}{|c|}{ Yield } \\
\hline (Reported in this & $\begin{array}{c}\text { (Reported } \\
\text { earlier) }\end{array}$ \\
\hline
\end{tabular}

\section{4: Unsaturation and Aromatization Reactions}

Unsaturated aldehydes, esters and lactones can be prepared by the process of free radical bromination followed by elimination. ${ }^{30}$ The allylic bromination of unsaturated lactones followed by base give dienoic and trienoic lactones (eqn. 15, 16). ${ }^{31,32}$

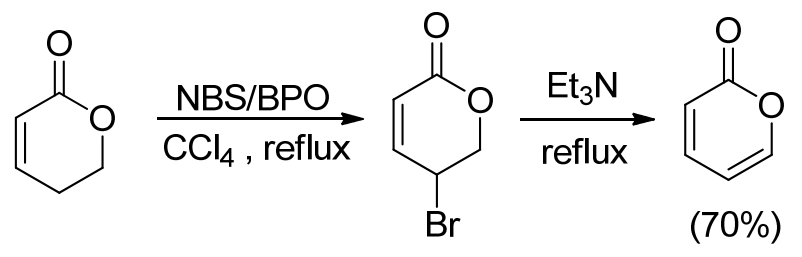




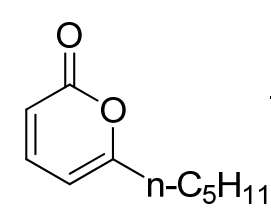

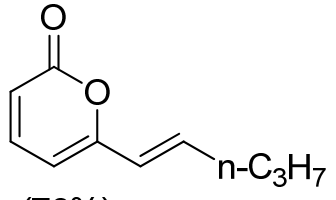

(70\%)

$\alpha, \beta$-unsaturated aldehydes can be obtained by converting aldehydes to enol acetates followed by the radical bromination at the $\mathrm{C}_{\beta}$ position to proceed and ester hydrolysis (eqn. 17). ${ }^{33}$

$$
\underset{\mathrm{R}=n-\mathrm{C}_{3} \mathrm{H}_{7}}{\mathrm{R}} \underset{\mathrm{OAc}}{\stackrel{1 . \mathrm{NBS}, \mathrm{AIBN}, \mathrm{CCl}_{4}}{2 . \mathrm{NaOH}, \mathrm{MeOH}} \mathrm{R}} \underset{(86 \%)}{\longrightarrow} \mathrm{CHO}
$$

\section{5: $\alpha$-Bromination of Carbonyl Derivatives}

A radical bromination reaction does not occur at $\alpha$-position in the case of simple carbonyl derivatives. Substitution by electron donating groups stabilizes the radical intermediates by the capto-dative effect ${ }^{34}$ and thus facilitates the substitution reaction. Protected glycine derivatives are easily brominated by refluxing with NBS and BPO in $\mathrm{CCl}_{4}$ to give $\alpha$ bromoglycine derivatives ${ }^{35,36}$ which are stable precursors for novel $\alpha$-amino acids (eqn. 18). ${ }^{37}$

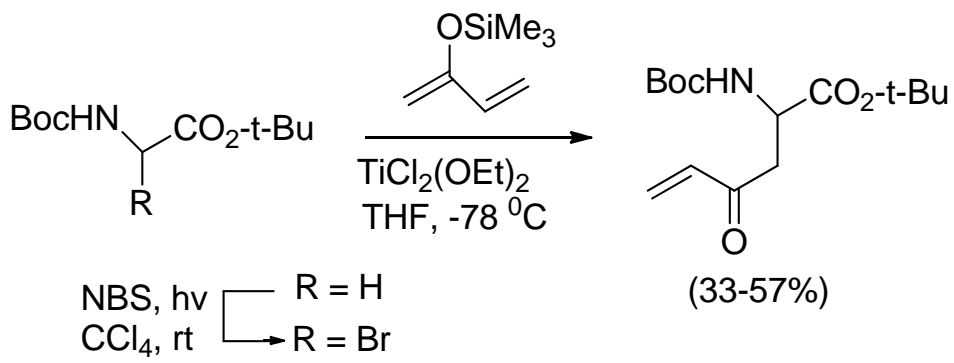

The use of NBS in the presence of catalytic $\mathrm{HBr}$ has proven to be more convenient than $\mathrm{Br}_{2}$ for conversion of acid chlorides to $\alpha$-bromo acid chlorides. ${ }^{38,39}$ The reaction of the corresponding enolates, enol ethers or enol acetates with NBS offers considerable advantages over direct acid-catalyzed halogenations of ketones and esters. ${ }^{40-42}$ Although both reagents may 
afford the $\alpha$-brominated products in high yields, NBS is more compatible than bromine with sensitive functional groups and has been used in the asymmetric synthesis of $\alpha$-amino acids. ${ }^{43,44}$ $o$-trimethylsilylaldoximes are readily brominated at the $\alpha$-position under radical bromination condition and can be converted to substituted nitrile oxides (o-trimethylslylketoximes react similarly). ${ }^{45}$

\section{6: Reaction with Vinylic and Alkynic Derivatives}

Vinyl copper and other organometallic derivatives can be converted to corresponding vinyl bromides by NBS. ${ }^{46}$ Reduction of 1-trimethylsilylalkynes with diisobytylaluminum hydride gives vinylsilanes which can be isomerized from the (Z) to the (E) geometry by irridation with NBS and Pyridine (eqn. 19). ${ }^{47,48}$

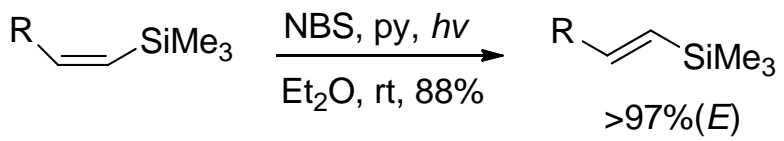

Allylsilane can be brominated by NBS under radical conditions, whereas more reactive allylsilanes are bromodesilated by NBS in $\mathrm{CH}_{2} \mathrm{Cl}_{2}$ at $-78^{0} \mathrm{C}$. ${ }^{49}, 50$ 1-bromoalkynes can be prepared under mild conditions by reaction with NBS in acetone in the presence of catalyst silver nitrate. ${ }^{51}$ Phenols, anilines and other electron rich aromatic compounds can be monobrominated on the ring using NBS in DMF (under electrophilic aromatic substitution condition) with higher yield and higher levels of para selectivity than with $\mathrm{Br}_{2}{ }^{52} \mathrm{~N}$-Trimethylsilylanilines and aromatic ethers are selectively brominated by NBS in $\mathrm{CCl}_{4}{ }^{53}$

Free radical bromination of 2, 3-dimethylanisole (20) with NBS shows highly regioselective character ${ }^{54}$. The intermediate monobrominated product obtained from the reaction of (20) and NBS in $\mathrm{CCl}_{4}$ eventually gets dibrominated. X-ray analysis revealed and reported that the Dibrominated product is (22) rather than (21). Further bromination of (22) with NBS in presence of $\mathrm{BPO}$ in $\mathrm{CCl}_{4}$ gave product (23) regiosepecifically. Alternatively, this was also obtained by the reaction of (20) with 3 equivalents of NBS. HNMR analysis revealed that ring brominated product (24) was obtained initially. GC/MS data showed that formation of (24) accompanied by parallel formation of its isomer (10\%). Eventually (24) and its isomer converted to (22), i.e.; the impurities do not affect the selectivity for the formation of (22). The probable 
isomeric product is (25). The reaction of (20) with NBS (1eqv.) under the same conditions but without a radical initiator lowered the rate significantly to give the ring brominated product (24) contaminated with small amount of (25).<smiles>COc1cccc(C)c1C</smiles>

(20)<smiles>COc1cccc(CBr)c1CBr</smiles>

(21)<smiles>COc1cccc(C)c1CBr</smiles><smiles>COc1ccc(Br)c(CBr)c1CBr</smiles>

(23)

The selective bromination of 3,4-dimethoxytoluene (26) with NBS was reported. The nuclear and side chain brominated products were obtained under different reaction conditions. ${ }^{55}$ The reaction of (26) with NBS in the ratio 1:1 gave benzyl bromide (27) as dominant product but unsatisfactory yield was reported. Dibrominated product (29) was reported to be obtained when 2:1 ratio of the reactants was used. It is reported that the use of BPO shortened the reaction time but does not increase the yield. Same reaction at room temperature or in polar solvent give nuclear brominated product (28).<smiles>COc1ccc(C)cc1OC</smiles>

(26)<smiles>COc1cc(C)c(Br)cc1OC</smiles>

(27)
(28)<smiles>COc1cc(Br)c(CBr)cc1OC</smiles>

(29) 
The presence of methoxy group is a prerequisite for aromatic bromination was reported earlier. $^{32,54,56}$ The methoxy group activates the aromatic ring at the $p$-position. The side chain bromination follows a radical pathway, while aromatic bromination is an electrophilic substitution reaction (Scheme 2). Low temperature and polar solvent facilitate aromatic bromination while high temperature and non-polar solvent facilitate free radical bromination.

Scheme 2: Nuclear Bromination mechanism by NBS

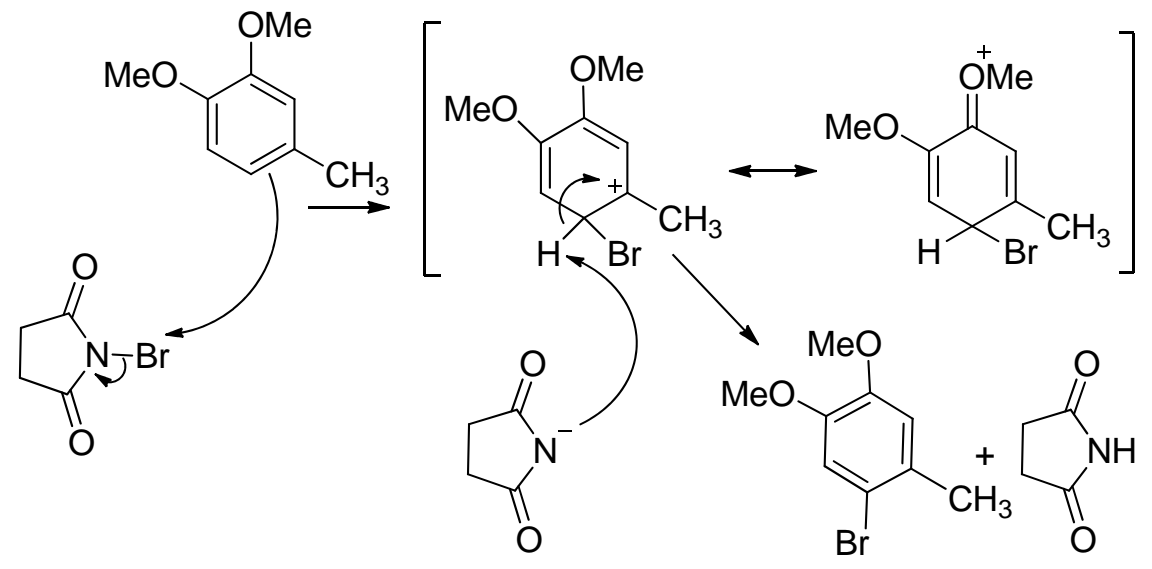

The evidence shows that the replacement of allylic or benzylic hydrogen by bromine from NBS proceeds through a free radical chain mechanism. ${ }^{57}$ The allylic and benzylic bromination by NBS is apparently analogous. ${ }^{9}$ Bloomfield proposed the involvement of succinimidyl radicals in the chain propagating steps in $1944 .^{58}$

A large body of evidence exists that bromination by NBS normally involves the bromine atom as the hydrogen-abstracting species rather than the $N$-succinimidyl radical. Goldfinger on his work on allylic chlorination with NCS has suggested halogen atom rather than succinimidyl radical may function as the chain carrying species as well. He has developed kinetic argument to show that low chlorine concentration should favor substitution, rather than addition. The halogen presumably is maintained in a low, constant concentration by an ionic reaction of $\mathrm{HCl}$ and NCS.

The most convincing evidence for the benzylic bromination mechanism involving bromine atom was advanced from studies on selectivites in the competitive brominations of mixture of substituted toluene with molecular bromine, $\mathrm{N}$-bromosuccinimide, $\mathrm{N}$-bromotetrafluorosuccinimide, and $N$-bromotetramethylsuccinimide. ${ }^{59}$ By studying six substituents they 
found an excellent correlation with $\sigma^{+}, \rho=-1.46, r=0.997$, thus suggesting a common reactive intermediate. ${ }^{60}$

The competitive bromination of series of a aralkyl hydrocarbons using molecular bromine in $\mathrm{CCl}_{4}$, NBS in $\mathrm{CH}_{2} \mathrm{Cl}_{2}$ and bromotrichloromethane in $\mathrm{CCl}_{4}$ as the brominating agents was studied. The similarity and uniqueness of relative reactivity series obtained for photo and NBS brominations indicated that the bromine atom is the hydrogen abstracting species in all reaction. ${ }^{61}$ Commercially available NBS is always slightly yellow due to the presence of traces of bromine and this could influence the stability of NBS. ${ }^{62}$ The small amount of bromine radical produced by NBS with radical initiator BPO initiates the main propagation steps. NBS thus has a double role (i) it provides a source of bromine, in low, steady state concentration and (ii) consumes the liberated $\mathrm{HBr}$ by an ionic process. ${ }^{63}$ The consumption of $\mathrm{HBr}$ avoids the inhibition of bromination. ${ }^{64}$

The predicted three step free radical mechanism for side chain bromination of 2-picoline with NBS is as shown in scheme 3.

Scheme 3: Free Radical Bromination mechanism by NBS

Step 1: Chain initiation<smiles>COOC(=O)c1ccccc1C(=O)OCOC(=O)c1ccccc1</smiles>

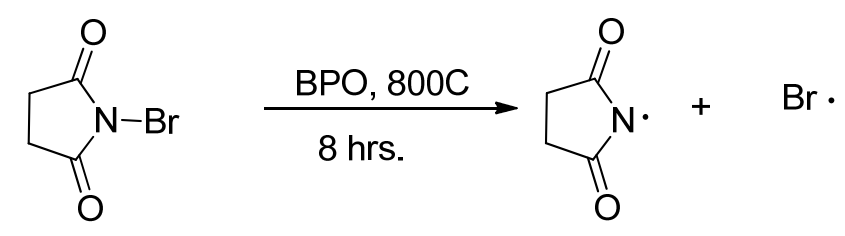


Step 2: Chain propagation<smiles>Cc1ccccn1</smiles><smiles>Cc1ccccn1</smiles>

Step 3: Chain termination

$\mathrm{Br} \cdot+\mathrm{Br} \cdot \longrightarrow \mathrm{Br}_{2}$<smiles>Cc1ccc[n+](Br)c1</smiles>

\section{7: Free Radical Bromination of Oligopyridine Benzylic-methyl Group}

Influence of three solvents on the photobromination of picolines was studied.

Dichloromethane and benzene were reported as better solvents than the classical solvent carbon tetrachloride. Bromination of toluene type model substrates using NBS and several solvents showed the strong influence of the solvent on the selectivity of the reaction ${ }^{65,66}$ and correlated to the reactivity-selectivity principle. ${ }^{67}$ The classical condition for the free radical bromination $\left(\mathrm{NBS} / \mathrm{CCl}_{4}\right)$ of the benzylic methyl group is not optimum due to the high reactivity, resulting a low selectivity. 
Scheme 4: Free Radical Bromination of Benzylic Methyl group<smiles>Cc1ccc(Br)nc1</smiles>

Two models of picolines bearing a benzylic methyl on the meta and ortho position of $\mathrm{N}$ in pyridine were selected (Scheme 4). Free radical bromination of 2-bromo-5-methylpyridine (30) and 2-bromo-6-methylpyridine (31) under several experimental conditions were reported (Table 2). The yield of products obtained by the reaction of one equivalent of picoline and same equivalent of bromine donor was compared.

\section{8: Solvents for NBS bromination}

Most commonly two solvents have been compared to $\mathrm{CCl}_{4}$ : dichloromethane and benzene. Methyl formate has been described as the solvent inducing the highest 'selectivity', in the case of toluene family but was reported to be ineffective with picolines, due to its lower reactivity. $\mathrm{CCl}_{4}$ was reported as the 'highest reactivity' solvent with a large amount of dibrominated product. (Table 2) For brominations of (30), benzene is reported to be most appropriate method in order to obtain (32) with minimum of (33). Due to the toxicity of $\mathrm{CCl}_{4}$ and benzene, $\mathrm{CH}_{2} \mathrm{Cl}_{2}$ was considered as the best solvent for this reaction. 
Table 2: Bromination of (30) with NBS using different solvents.

\begin{tabular}{|c|c|c|c|}
\hline Products & $\mathbf{C C l}_{\mathbf{4}}$ & $\mathbf{C H}_{\mathbf{2}} \mathbf{C l}_{\mathbf{2}}$ & $\mathbf{C}_{\mathbf{6}} \mathbf{H}_{\mathbf{6}}$ \\
\hline $30(\%)^{\mathrm{a}}$ & 12 & 19 & 24 \\
\hline $32(\%)^{\mathrm{a}}$ & 66 & 69 & 67 \\
\hline $33(\%)^{\mathrm{a}}$ & 22 & 12 & 9 \\
\hline
\end{tabular}

${ }^{\mathrm{a}}$ Relative yield calculated from ${ }^{1} \mathrm{H}$ NMR spectra

Different reactivity of hydrogen abstracting species was confirmed by the low yields of production of dibromomethyl compounds in case of benzene as opposed to $\mathrm{CCl}_{4}$, both having similar boiling points. In the presence of benzene, a bromine radical/arene $\pi$-complex is probably formed, similar to the complex observed with chlorine. ${ }^{68,69}$

Water was described as an excellent medium for free radical reaction, ${ }^{70}$ due to its remarkable non-reactivity towards radicals $(\mathrm{OH}$ bond resistancy to homolytic bond breaking).Unfortunately, the solubility of most organic compounds is limited in water. The photobromination of toluene with good results using bromine in water was reported. ${ }^{71}$ In fact, toluene acts as its own solvent and consequently the reaction is operated in a biphasic medium. The use of aqueous biphasic mixture of solvent and bromine has been reported to be a convenient synthetic procedure for the free-radical bromination of selected compounds.

The study of the free radical bromination of (31) with NBS in the biphasic mixture was reported which shows similar reactivity to the monophasic case. The yield difference between the bromination of (30) and (31) are enhanced with the use of bromine. Under these conditions, it was anticipated that presence of lone pair of electron in nitrogen has a significant action on the free radical process.

Radical mediated bromination is frequently used to achieve selective activation of methyl group in appropriate organic molecule. The free radical bromination reaction is normally carried out by using NBS in $\mathrm{CCl}_{4}$ with various radical initiators. ${ }^{71}$. $\mathrm{CCl}_{4}$ solvent is restricted due to its high toxicity, including possibly carcinogenicity, which restricts its use. $\mathrm{CCl}_{4}$ can be replaced by other solvents e.g. methyl acetate, in light and microwave assisted benzylic brominations. ${ }^{72}$ NBS in (trifluoromethyl) benzene ( $\alpha, \alpha, \alpha$-trifluorotoluene, benzotrifluoride) was used as a solvent for 
benzylic bromination because of its favorable physical properties, chemical inertness and low toxicity. ${ }^{73}$

The efficient bromination reaction of mono, di, or as trimethylated aromatic and heterocyclic system under photochemical conditions with NBS in the presence of AIBN, or BPO as radical initiator in (trifluoromethyl) benzene was reported. (Trifluoromethyl) benzene was chosen as solvent not only because of its inertness and low toxicity, but also on account of its low refractive index, an important property for achieving efficient photo-induced bromination of NBS. ${ }^{66}$

Effective monobromination of each of two or more methyl group attached to a benzenoid ring was reported in a project aimed for the preparation of artificial receptors. ${ }^{74}$ The important target molecules were the tribromides 1-\{1,1-bis[4-(bromomethyl)phenyl] ethyl $\}$-4(bromomethyl)benzene (36a) and 1-\{1,1-bis[4-(bromomethyl)phenyl\}ethyl\}-3-(bromomethyl) benzene. (37b) derived from 1-[1,1-bis(3-methyl)ethyl]-4-methylbenzene (36) and its isomer 1[1,1-bis(4-methylpheynyl)ethyl]-3-methyl benzene (37). Benzylic bromination of methyl groups of (36) and (37) were reported to explore expecting that it would be difficult to avoid polybromination.

Bromination of mono-, di- or trimethylated aromatic and heterocyclic systems were reported to occur efficiently under photochemical conditions with NBS in the presence of 2,2'azobisisobutyronitril (AIBN), 1,1'-azobis(cyclohexanecarbonitrile) (ACCN) or benzoyl peroxide (BPO) as radical initiator in (trifluoromethyl)benzene.

The trimethyl substrates (36) and (37) and the dimethyl substrate reported to provide a good test of the strength of the procedure because of the opportunities for polybromination. ${ }^{74}$ The procedure developed reported to give acceptable yields of the desired product (36)-(38) (Table 3). As expected, no detectable bromination of the non benzylic methyl group in compounds (36) and (37) were observed. Mono- and dimethyl aromatic compounds and mono- and dimethyl heterocyclic compounds were also reported to give good yields (Table 3), which are comparable to the best results in the literature. ${ }^{65,75-79}$ Variants of the classical NBS procedures using bromine or bromine-generating systems in the presence of water. ${ }^{71,75}$ Bromination (42) was reported to be accomplished efficiently by using bromine in a two-phase $1: 1(\mathrm{v} / \mathrm{v})$ mixture of water and (trifluoromethyl) benzene. 
Table 3: Benzylic bromination by NBS in (trifluoromethyl) benzene

(2)




\begin{tabular}{|l|l|l|l|}
\hline & 6 & \\
\hline
\end{tabular}

\section{9: NBS under Solvent Free Conditions}

The visible light-induced reaction of toluene with NBS under solvent free conditions (SFRC) was studied. ${ }^{62}$ In the scope of green chemistry "the best solvent is no solvent” examples of solvent free fluorination, chlorination, iodination and bromination are reported in both old and recent literature. Selective radical chain reactions were reported to carry out in liquid/liquid and in solid/solid systems. Toluene is regioselectively converted to benzyl bromides. The reaction scheme follows

Scheme 5: NBS Bromination of Toluene and substituted Toluene<smiles>[R]c1ccc(C)cc1</smiles>

The radical scavenger 2,2,6,6-tetramethylpiperdin-1-yloxy (TEMPO) completely suppressed the side chain bromination of toluene with NBS under SFRC was reported. The reactivity of toluene was found to depend on the substituent; ERG was reported to increase whereas EWG decrease the reactivity. Despite being a heterogeneous reaction system, a good Hammett free-energy relationship was reported. Hammet reaction constant $\rho^{+}=-1.7$ indicated involvement of polar radical intermediate with electrophilic character in the radical reaction under SFRC. 


\subsection{0: Regioselective bromination of Polymethylpyrimidines}

Direct bromination of polymethylbenzene has been widely used in the synthesis of bromomethyl derivatives. ${ }^{65}$ Few efficient preparations of bromo methyl-substituted azaaromatic compounds via direct bromination have been reported because overbromination is the major problem. Many successful preparations of tribromomethyl-substituted pyridines, ${ }^{80}$ pyrimidines, ${ }^{81}$ quinolines, ${ }^{82}$ phenanthridines, ${ }^{82}$ and phenthrolines ${ }^{83}$ from corresponding methyl derivatives are reported. Regioselective brominations of 2,3-dimethylpyridine ${ }^{84}$ with 1 equivalent of NBS have been reported to give mainly a 3-(bromomethyl )-substituted product, though in a low overall yield. A similar regioselectivity was observed in the reaction of 4,6-dichloro-2,5-dimethylpyrimidine with NBS, which gave a 5-(bromomethyl)-pyrimidine. Opposite regioselectivity of the bromination reaction of ethyl 6-hydroxy-2,5-dimethyl-4-pyrimidinecarboxylate with bromine in acetic acid to give a 2-tribromomethyl derivative in a high yield. ${ }^{81}$ These examples suggest that the nitrogen atom in the ring plays a pivotal role in regioselective brominations of methyl substituents with the two reagents. While the methyl group located meta to the ring nitrogen is brominated selectively with NBS, the ortho methyl group undergoes selective bromination with $\mathrm{Br}_{2} / \mathrm{AcOH}$ was reported.

Scheme 6: Selective Bromination with $\mathrm{Br}_{2} / \mathrm{AcOH}$

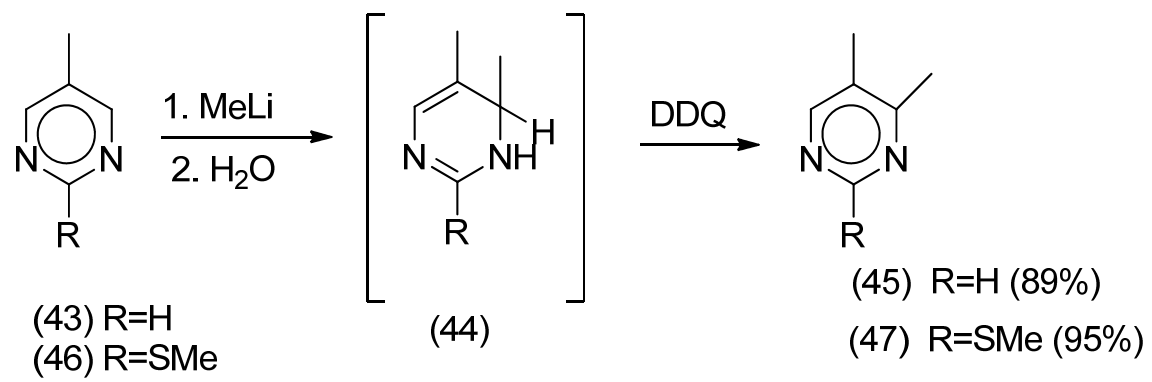




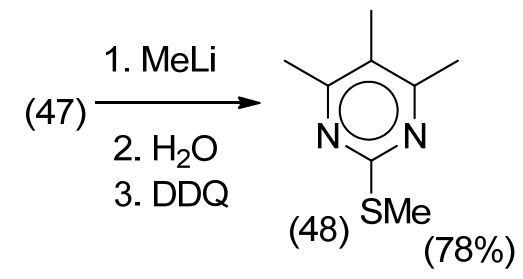

Bromination of dimethylpyrimidines (45) and (47) and a sterically congested trimethylpyrimidine (48) were reported. ${ }^{85}$ Selective introductions of bromine atoms at benzylic positions of these pyrimidines were anticipated to provide an invaluable intermediate for a facile modification of the pyrimidine ring.

A new synthesis of pyrimidines (45), (47) and (48) is given in above scheme 6 . The synthesis scheme is based on the addition reaction of methyllithium with a formal $\mathrm{C}=\mathrm{N}$ double bond resultant dihydropyrimidine (44), without isolation, by treatment with DDQ (2,3-dichloro5,6-dicyanobenzoquinone). ${ }^{86}$ This method is superior to other reported preparations of $(45)^{87}$, $(47)^{88}$ and (48) $)^{89}$ because of its simplicity and high efficiency.

The reaction of (45) with NBS (Scheme 6) is reported to give unstable 5-(bromomethyl)pyrimidine (50) and a stable 5-(dibromomethyl)pyrimidine (49) as a major and minor product, respectively. The pure sample of both products were obtained by chromatographic separation and characterized by NMR, including NOE experiments to establish the bromination site, and by MS. Treatment of (45) with $\mathrm{Br}_{2} / \mathrm{AcOH}$ reported to produced unstable 4-(bromomethyl) pyrimidine (51) as the major product and stable 4-(dibromomethyl)pyridine (52). As with (45), the opposite high regioselectivities with similar ratios of bromomethyl- to dibromomethylsubstituted product were reported to observe in the reaction of (47) with NBS and bromine. 
Scheme 7: Bromination of Dimethyl pyridines<smiles>[R]c1ncc(C(Br)Br)c(C)[nH+]1</smiles>

(49) $\mathrm{R}=\mathrm{H}(10 \%)$

(53) $\mathrm{R}=\mathrm{SMl}(15 \%)$

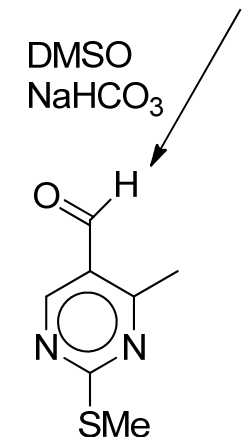

(57)

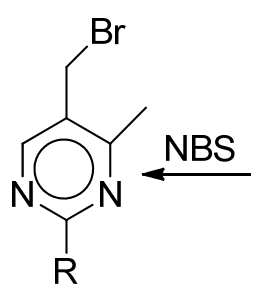

(50) $\mathrm{R}=\mathrm{H}(77 \%)$

(54) $\mathrm{R}=\mathrm{SMe}(68 \%)$

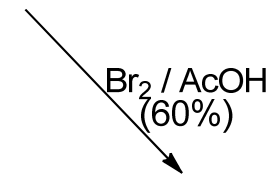

$\checkmark$<smiles>COc1ncc(CBr)c(CBr)n1</smiles>

(45), (47)<smiles></smiles>

(51) $\mathrm{R}=\mathrm{H}(75 \%)$

(55) $\mathrm{R}=\mathrm{SMe}(81 \%)$<smiles>[R]c1ncc(C)c(C(Br)Br)n1</smiles>

(52) $\mathrm{R}=\mathrm{H}(6 \%)$

(56) $\mathrm{R}=\mathrm{SMe}(5 \%)$

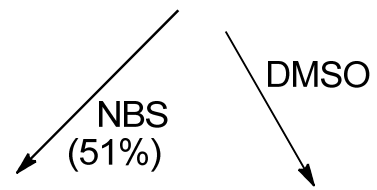<smiles>CSc1ncc(C)c(C=O)n1</smiles>

(59)

The bromination reaction of (48) followed the patterns of those regioselectivity observed with (45) and (47) reported to give the corresponding bromomethyl derivatives in high yields ${ }^{85}$ (scheme 7). Compound (60) with NBS and (62) with $\mathrm{Br}_{2} / \mathrm{AcOH}$ were reported to produce tris(bromomethyl)pyrimidine (63). Dibromomethyl derivatives are apparently not formed due to steric hindrance in (63) was reported.

Scheme 8: Bromination of compound (48)<smiles>CSc1nc(C)c(C)c(C)n1</smiles>

(48)<smiles>CSc1nc(CBr)c(CBr)c(CBr)n1</smiles>

(60) $(82 \%)$<smiles>CSc1nc(CBr)c(C)c(C(Br)Br)n1</smiles>

(61) (4\%) 
<smiles>Cc1nc(SCC(C)C)nc(C)c1C</smiles>

(48)<smiles>CSc1nc(C)c(CBr)c(CBr)n1</smiles>

(62)<smiles>CSc1nc(CBr)c(CBr)c(CBr)n1</smiles>

(63)

The bromination reaction of non hindered methylpyrimidine (64) with $\mathrm{Br}_{2} / \mathrm{AcOH}$ was reported to give dibromomethyl and bromomethyl derivatives (65) and (66) as major products. (Scheme 8) Electrophilic bromination of the pyrimidine ring is also reported to occur as the minor product (67). When the reaction was conducted in the presence of sodium acetate, sulfoxide (68) and sulfone (69) were reported. (68) could be converted to (69) using the same condition. This results suggest that benzylic bromination of 4-methylpyrimidine with $\mathrm{Br}_{2}$ and AcOH involves protonation of pyrimidine ring. This protonation is suppressed in a mixture of AcOH with AcONa, which is less acidic than AcOH itself.

Scheme 9: Bromination of Non-hindered Methyl Pyridine<smiles>CSc1ncc(CBr)c(CBr)n1</smiles>

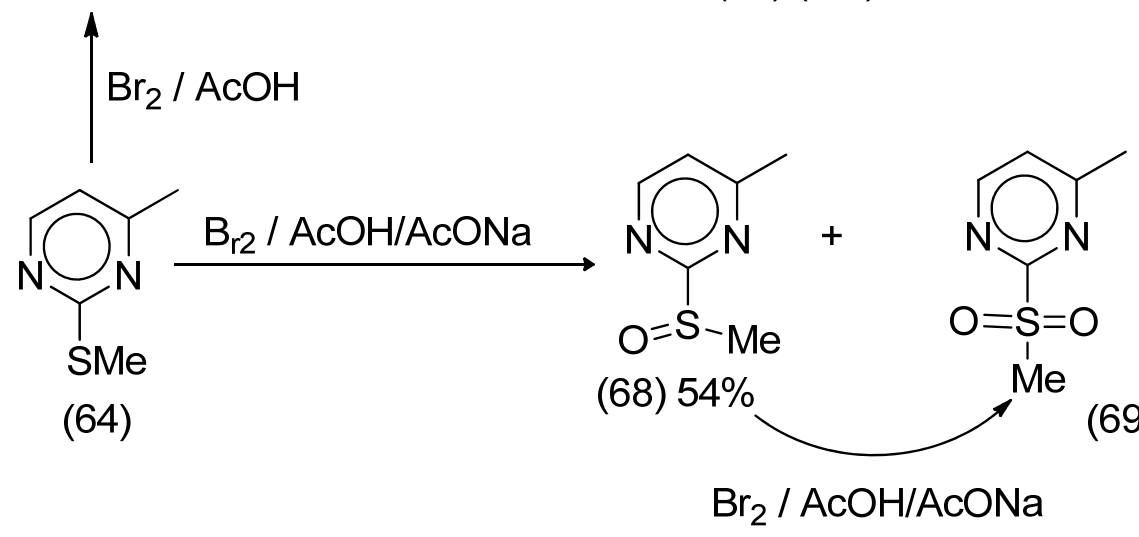

(69) $16 \%$ 


\subsection{1: An Unusual Orienting effect in the Bromination of Alkyl Bromides}

The directive influence of polar substituents in free radical halogenation reaction has been studied by several groups. ${ }^{90,91}$ These studies discovered that halogenation occur preferentially at positions removed from the electron withdrawing substituent (EWS). The results are consistent with theory that the electronegative attacking radicals prefer positions of higher electron density.

The halogenation of several alkyl and cycloalkyl halides is through photo- bromination of alkyl bromides is quite different from the bromination of other alkyl halides and from alkyl halide halogenations in general. ${ }^{92}$ The chlorination of alkyl bromides is reported to produce the expected isomer distribution; preferential attack occurring at positions remote from the bromine substituent. Bromination of alkyl chloride also showed the usual polar orientating effect.

Unlike other free radical halogenation of alkyl halide which frequently give a multitude of products, the bromination of alkyl bromides was reported as highly selective, giving the vicinal dibromide isomer (84-94\%). The influence of the bromine substituent directing the attack of a halogen atom to the adjacent carbon is contrary to other radical halogenations of substituted alkanes, in which this position has been demonstrated, generally, to be one of the least reactive positions in the molecule. A neighboring group effect has been postulated to explain the observed results.

The reports of bromination of different compounds are summarized as follows:

a. Bromination of Bromocyclohexane.

Photobromination of bromocyclohexane is reported to give trans-1,2-dibromocyclohexane by comparison with infrared spectrum. ${ }^{93}$ The appearance of only one out of seven isomeric dibromocyclohexanes suggested that perhaps this was not a free radical process but instead involved the elimination of $\mathrm{HBr}$ from bromocyclohexane, followed by the ionic addition of bromine to cyclohexene to give trans-1,2-cyclohexane.

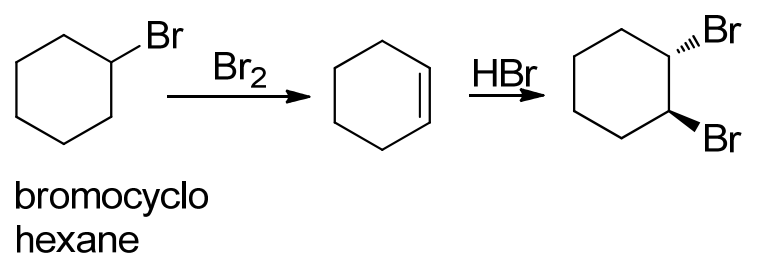




\section{b. Bromination of Bromocyclopentane}

Photobromination of bromocyclopentane was reported to give trans-1,2-dibromocyclopentane. This was confirmed by infrared spectrum, refractive index and vapor phase chromatographic analysis.

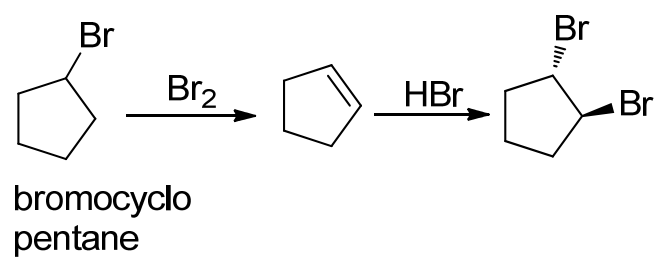

c. Bromination of Chlorocyclohexane.

Bromination of chlorocyclohexane was investigated ${ }^{92}$ in order to determine the importance of the bromine substituent in direction the substitution of bromine to carbon two. Preparation of trans-1-bromo-2-chlorocyclohexane was by the action of NBS and $\mathrm{HCl}$ on cyclohexene. ${ }^{94}$ with comparision it was reported that one to the product matches with this compound.

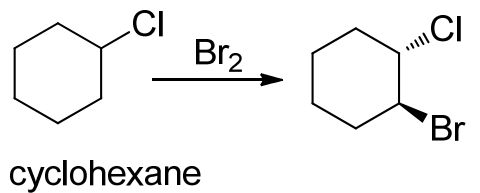

\section{d. Bromination of 1-Bromobutane}

The vapor phase bromination of some alkyl halides was investigated by Kharasch; ${ }^{90}$ the halogenation of butyl halides in general, more recently by Tedder and Fredricks. Kharasch reported that the bromination of alkyl bromides in general led to the formation of large amounts of tri- and tetrasubstituted bromides. On the other hand, Tedder reported that this bromination did not proceed well; large portions of the bromine remained unreacted and a brown coating formed on the surface for the reaction vessel. The liquid phase bromination of 1-bromobutane was reported to give isomers of dibromobutane. The major products are reported 1,2- and the 1,3-dibromobutanes. ${ }^{92}$ 


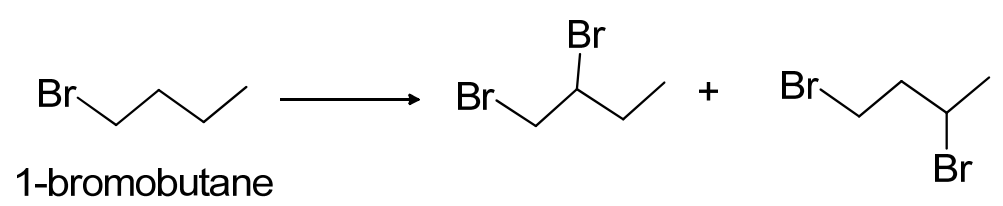

e. Bromination of 1- Chlorobutane

The bromination of 1-chlorobutane was reported and The directing influence of chlorine to that of bromine was reported. The v.p.c. analysis of the reaction mixture showed the formation of three major products which are 1,1-, 1,2-, and 1,3-chlorobromobutanes in the ration 1:1:2. This isomer distribution was reported the same as those found by Kharasch. ${ }^{90}$ Hence, the conclusion was made that the bromination of n-butyl chloride and cyclohexyl chloride is quite different than corresponding bromides and does not occur preferentially at carbon two.

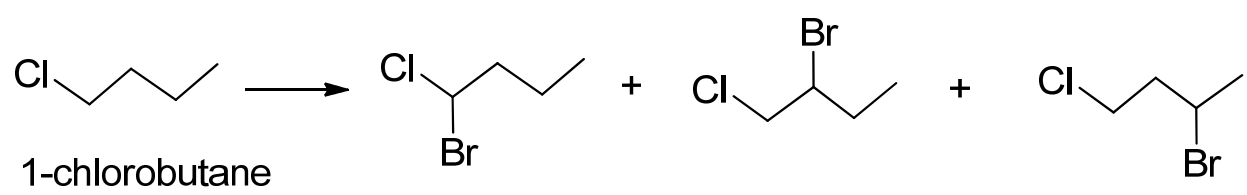

\section{f. Bromination of 2-Bromobutane}

Bromination of 2bromobutane is reported to give three products, i.e.; 2,2- dibromobutane, meso2,3-dibromobutane, and $d l$-2,3-dibromobutane. The 2,3-dibromobutanes comprised over $83 \%$ of the products, these again demonstrates the strong orientating influence toward the $\alpha$-carbon atom which the bromine substituent exerts.

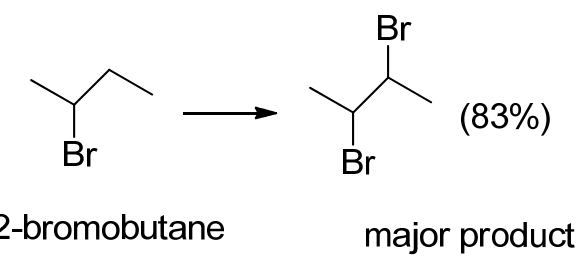

g. Bromination of 2-Chlorobutane

Vapor phase chromatographic analysis of the reaction mixture reported to reveal a three peaks pattern similar to that observe from the 2-bromobutane bromination. The three peaks are 
found to be the 2,2-chlorobromobutane, erythro- and threo-2,3-dihalobutanes . Two major factors on free radical halogenation of hydrocarbon and derivatives that influence the distribution of possible isomers were discussed, C-H bond energies and inductive effect.

The halogenation of parafins provides an example of $\mathrm{C}-\mathrm{H}$ bond energy. Hydrogens are substituted in the order of tertiary > secondary > primary, which is the order of increasing bond energies. The selectivity between these hydrogens becomes increasingly greater as the activation energy of the abstracting process increases. This large difference in activation energy is the determining factor for the marked selectivity of bromination and the relatively poor selectivity of chlorination.

The presence of electronegative groups on a molecule introduces an additional consideration. Halogen atoms are electronegative (having tendencies to pull electron towards themselves) and prefer to attack positions of higher electron density, which is due to the inductive influence of the substituent. In addition, the substituent also increases the polarity of the $\mathrm{C}-\mathrm{H}$ bond to which it is attached hence lowers the bond energy. Higher the polarity lower will be bond energy and higher will be the reactivity.

In free radical chlorination and even more in fluorination, the polar effect predominates and reactivity at the substituent bearing carbon is low (because the availability of electron is low). In higher activation energy bromination process, the influence of bond energy is much more important and substituent bearing carbon is significantly more reactive than in chlorination.

\subsection{2: Electronic effect in selectivity $\sigma^{+}$values for different position of pyridine}

The application of the Hammett equation for the prediction of the effects of substituents on the heterocyclic compounds was reported. ${ }^{95}$ The derived $\sigma^{+}$values have proven useful in correlating on electrophilic aromatic substitution and electrophilic side-chain reaction. ${ }^{96}$ Free radical bromination of toluene with different brominating agents was carried out. The rates relative to toluene for hydrogen abstraction from substituted toluene by various brominating agents were accumulated and average $\rho$ value of -1.42 was obtained with nearly independent of the brominating agents. In all cases, an excellent fit of the points to the Hammet correlation was obtained when $\sigma^{+}$values were used. ${ }^{59}$ The published $\sigma$ values for methyl pyridines are as follows: 


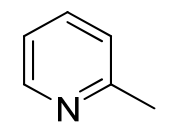

$\sigma=0.81$

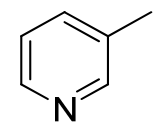

$\sigma=0.62$

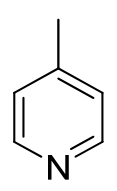

$\sigma=0.93$

Extending this work to picolines, the substituents effects i.e.; $\sigma$ values of 2-picoline and 4-picoline show that the reactivities of 2-picoline and 4-picoline should be similar whereas the reactivity of 3-picoline seems higher compared to 2 and 4-picolines owing to the higher $\sigma$ value. $^{59}$ The order of reactivity of picoline comparing its $\sigma$ value can be given as 3-methyl $>2$ methyl > 4-methyl. Hasegawa had shown that 2-picoline reacts with NBS to give mixture of mono and di-substituted products. Similarly 4-picoline was reacted with NBS and the product was isolated as the hydrobromide salt. The analysis of the product revealed that the product was neither mono- nor di-bromomethylpyridine but the fully brominated, 4-tribromomethyl-pyridine.

Several attempts were made by Kutney et. al. (1964) to brominate the methyl group in 3methylpyridine under the same conditions but no bromo compounds could be obtained. On the basis of all the results, it was concluded that in the methylpyridine series, the rate of reactivity towards NBS is 4-methyl > 2-methyl > 3-methyl. ${ }^{80}$ The classical approach to explain reactivity in relation to $\sigma$ value seems inadequate.

\subsection{3: Free Radical Halogenation}

There are great differences in the reactions of other halogen relative to bromination. In the case of chlorination, although the same chain mechanism as for bromination is operative, there is a key difference in the greatly diminished selectivity of the chlorination. Because of the greater reactivity of the chlorine atom, abstractions of primary, secondary and tertiary hydrogens are all exothermic. As a result, the stability of the product radical has less influence on the activation energy. In term of Hammond postulate, the transition state is more reactant-like. As an example of the low selectivity, ethyl benzene is chlorinated at both the methyl and the methylene positions, despite the much greater stability of the benzyl radical. ${ }^{97}$ 


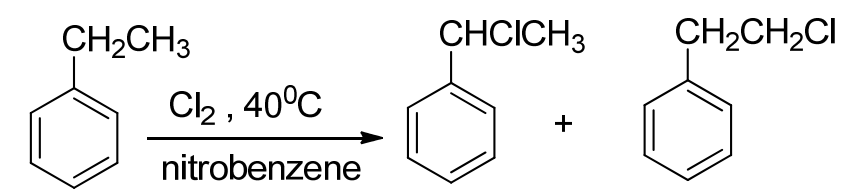

Radical chlorination reactions show a substantial polar effect. Positions substituted by EWG are relatively uncreative towards chlorination, even though the substituents may be potentially capable of stabilizing the free radical intermediates. ${ }^{10}$

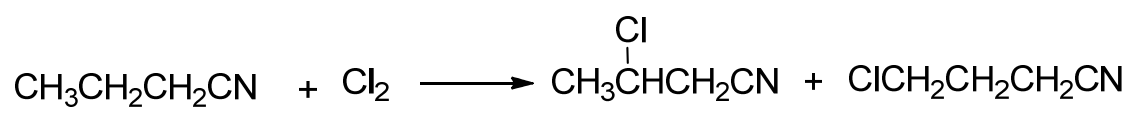

The polar effect is attributed to the fact that the chlorine atom is an electrophilic species, and the relatively electron-deficient carbon atoms adjacent to the EWGs are avoided. Carboxylic acid and ester groups tend to direct chlorination to the $\beta$ and $\gamma$ positions, because attack at the $\alpha$ position is electronically disfavored. The effect of a EWS is to decrease the electron density at the potential radical site, even though there would be a net stabilization of the radical that would be expected to have a very early transition state. The polar substituent effect dominates the kinetic selectivity of the reaction, and the stability of the radical intermediate has relatively little influence.

Radical substitution reactions by iodine are not practical because the abstraction of hydrogen from hydrocarbons by iodine is highly endothermic, even for stable radicals. The enthalpy of the overall reaction is also slightly endothermic. Thus, both because of the kinetic problem excluding a chain reaction and an unfavorable equilibrium constant for substitution, iodination cannot proceed by a radical chain mechanism.

Fluorination presents problems of the other extreme. Both steps in the substitution chain reaction are so exothermic that the reaction is violent if not performed under carefully controlled conditions. Furthermore, fluorine atoms are capable of cleaving carbon-carbon bonds:

$$
\mathrm{F}^{\cdot}+\mathrm{CH}_{3} \mathrm{CH}_{3} \longrightarrow \mathrm{CH}_{3} \mathrm{~F}+\mathrm{CH}_{3}^{\cdot}
$$

Saturated hydrocarbons such as neopentane, norborane, and cyclotane have been converted to the corresponding perfluoro derivatives in $10-20 \%$ yield by gas phase reaction with 
fluorine gas diluted with helium at $-78^{0} \mathrm{C} \cdot{ }^{98}$ Simple ethers can be completely fluorinated under similar conditions. ${ }^{99}$

Halogenations of organic molecules can be carried out with several other chemical reagents in addition to the molecular halogens. N-Bromosuccinimide (NBS) has been used extensively, especially for allylic and benzylic bromination.

\subsection{4: Chorination with $N$-Chlorosuccinimide}

Comparative evaluation of the free radical chlorination by $\mathrm{N}$-Chlorosuccinimide NCS for a series of heterocyclic system is reported. ${ }^{100}$ Free radical chlorination using NCS gives mixture of different multiple chlorine substituted products.

Mixture of Mono (25\%0 and di (20\%) chlorinated products are obtained on the free radical chlorination with of 2-picoline while only mono substituted products is obtained with 3picoline. Mixture of multi substituted products are obtained during the free radical chlorination of 2,4; 2,5 and 2,6-lutidines.

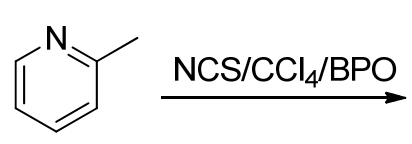

2-methyl pyridine<smiles>Cc1cccnc1</smiles>

3-methyl pyridine<smiles>Cc1ccnc(C)c1</smiles>

2,4-Lutidiene<smiles>ClCc1ccccn1</smiles>

$20 \%$

$25 \%$<smiles>ClCc1cccnc1</smiles>

$26 \%$<smiles>Cc1ccnc(CCl)c1</smiles>

$16 \%$<smiles>[13CH3]c1ccnc(C(Cl)(Cl)Cl)c1</smiles>

$13 \%$<smiles>ClCc1ccnc(C(Cl)(Cl)Cl)c1</smiles> 
<smiles>Cc1ccc(C)nc1</smiles><smiles>Cc1cccc(C)n1</smiles><smiles>ClCc1cccc(C(Cl)Cl)n1</smiles>

Elemental chlorine can be used in free-radical halogenation reactions, too, but these reactions are less easily controlled as the $\mathrm{Cl} \bullet$ radical is more reactive than the $\mathrm{Br} \bullet$ Radical and hence less selective.

\subsection{5: Selectivity of free-radical halogenation}

Mixture of methane and chlorine reacts in presence of light or heat to give chloromethane and hydrochloric acid with the evolution of heat. If chloromethane is allowed to react with more chlorine, mixtures of chloromethanes i.e.; multisubstituted chloromethanes will be obtained.

$$
\begin{aligned}
& \mathrm{CH}_{4}+\mathrm{Cl}_{2} \stackrel{\text { heat }}{\longrightarrow} \mathrm{CH}_{3} \mathrm{Cl}+\mathrm{HCl}-100 \mathrm{~kJ}(23.9 \mathrm{kcal}) / \mathrm{mol} \\
& \mathrm{CH}_{3} \mathrm{Cl}+\mathrm{Cl}_{2} \stackrel{\text { heat }}{\longrightarrow} \mathrm{CH}_{2} \mathrm{Cl}+\mathrm{HCl} \\
& \mathrm{CHCl}_{3} \stackrel{\mathrm{Cl}_{2}}{\longrightarrow} \mathrm{CH}_{2} \mathrm{Cl}_{2} \stackrel{\mathrm{Cl}_{2}}{\longrightarrow} \mathrm{CCl}_{4}
\end{aligned}
$$

Bromine reacts exactly the same way as chlorine; however, it is far more selective. If propane, for example, was the substrate, 2-bromopropane would be the dominant product, and there would be only a small amount of 1-bromopropane. Free radical chlorination, though, would not be quite as selective, and there would be a greater amount of the chlorination of the primary carbon than in the bromination reaction. 


$$
\begin{aligned}
& \mathrm{CH}_{3} \mathrm{CH}_{2} \mathrm{CH}_{3}+\mathrm{Br}_{2} \stackrel{\text { heat or light }}{\longrightarrow} \mathrm{CH}_{3} \stackrel{\mathrm{Br}}{\mathrm{C}} \mathrm{HCH}_{3}+\mathrm{CH}_{3} \mathrm{CH}_{2} \mathrm{CH}_{2} \mathrm{Br}+\mathrm{HBr} \\
& \text { 2-Bromopropane 1-Bromopropane } \\
& (92 \%) \quad(8 \%) \\
& \mathrm{CH}_{3} \mathrm{CH}_{2} \mathrm{CH}_{3}+\mathrm{Cl}_{2} \stackrel{\text { heat or light }}{\longrightarrow} \underset{\stackrel{\mathrm{Cl}}{\mathrm{l}}}{\mathrm{CH}_{3}} \mathrm{HCH}_{3}+\mathrm{CH}_{3} \mathrm{CH}_{2} \mathrm{CH}_{2} \mathrm{Cl}+\mathrm{HCl} \\
& \text { 2-Chloropropane 1-Chloropropane } \\
& (57 \%) \quad(43 \%)
\end{aligned}
$$

The reaction occurs by a free-radical mechanism in which the first (initiation) step involves the homolytic cleavage of halogen molecules to give halogen atoms. This cleavage can be promoted by either heat or light. This is then followed by a "chain” of propagation steps, which are shown below.

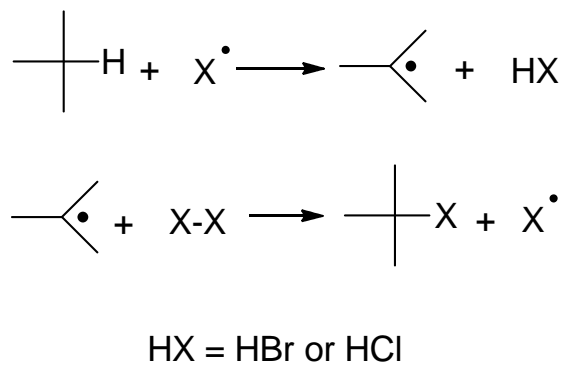

The halogen atom abstracts a hydrogen atom from the substrate, and the overall rate of the process is determined by the stability of the carbon radical formed. This radical then reacts with molecular halogen to give the product and regenerate a halogen atom to carry on the chain process. In the free radical processes, the intermediates are so highly reactive and short lived that usually mixture of products are obtained, even though there is preference for forming more highly substituted free radical intermediates.

The order of regioselectivity of halogenation of aliphatic alkane follows the order $3^{0}>2^{0}$ $>1^{0}$. Regioselectivity for bromination is higher than for chlorination. Regioselectivity for halogenation is in the order of $3^{0}>2^{0}>1^{0}$ which is approximately1600:80:1 for bromination and is approximately 5:4:1 for chlorination. 
Table 4: Bond Dissociation Enthalpies for hydrocarbons

\begin{tabular}{|l|l|l|l|c|}
\hline Hydrocarbon & \multicolumn{1}{|c|}{ Radical } & \multicolumn{1}{|c|}{$\begin{array}{c}\text { Name of } \\
\text { Radical }\end{array}$} & \multicolumn{1}{|c|}{$\begin{array}{c}\text { Type of } \\
\text { Radical }\end{array}$} & $\begin{array}{c}\Delta \mathbf{H}^{\mathbf{0}} \\
(\mathbf{k J})\end{array}$ \\
\hline $\mathrm{CH}_{2}=\mathrm{CHCH}_{2}-\mathrm{H}$ & $\mathrm{CH}_{2}=\mathrm{CHCH}_{2} \bullet$ & Allyl & Allylic & 372 \\
\hline $\mathrm{C}_{6} \mathrm{H}_{5} \mathrm{CH}_{2}-\mathrm{H}$ & $\mathrm{C}_{6} \mathrm{H}_{5} \mathrm{CH}_{2} \bullet$ & Benzyl & Benzylic & 376 \\
\hline$\left(\mathrm{CH}_{3}\right)_{3} \mathrm{C}-\mathrm{H}$ & $\left(\mathrm{CH}_{3}\right)_{3} \mathrm{C}^{\bullet}$ & tert-Butyl & tertiary $\left(3^{0}\right)$ & 405 \\
\hline$\left(\mathrm{CH}_{3}\right)_{2} \mathrm{CH}-\mathrm{H}$ & $\left(\mathrm{CH}_{3}\right)_{2} \mathrm{CH} \bullet$ & Isopropyl & secondary $\left(2^{0}\right)$ & 414 \\
\hline $\mathrm{CH}_{3} \mathrm{CH}_{2}-\mathrm{H}$ & $\mathrm{CH}_{3} \mathrm{CH}_{2} \bullet$ & Ethyl & primary $\left(1^{0}\right)$ & 421 \\
\hline $\mathrm{CH}_{3}-\mathrm{H}$ & $\mathrm{CH}_{3} \bullet$ & Methyl & Methyl & 439 \\
\hline $\mathrm{CH}_{2}=\mathrm{CH}-\mathrm{H}$ & $\mathrm{CH}_{2}=\mathrm{CH} \bullet$ & Vinyl & Vinylic & 464 \\
\hline
\end{tabular}

The heat of reaction $\left(\Delta \mathrm{H}^{0}\right)$ can be calculated by using bond dissociation values (BDE) for the halogenation of alkanes as follows.

\begin{tabular}{lllll} 
& \multicolumn{2}{c}{$\mathrm{CH}_{4}+\mathrm{Cl}_{2} \stackrel{\text { heat }}{\longrightarrow}$} & $\mathrm{CH}_{3} \mathrm{Cl}+\mathrm{HCl}$ & $\Delta \mathrm{H}^{0}=-96 \mathrm{~kJ}$ \\
$\mathrm{BDE}, \mathrm{kJ} / \mathrm{mol}$ & $+439+247$ & -351 & -431 & $(23.9 \mathrm{kcal}) / \mathrm{mol}$ \\
$(\mathrm{kcal} / \mathrm{mol})$ & $(+105)(+59)$ & $(-84)$ & $(-103)$ &
\end{tabular}

The regioselectivity of bromination and chlorination can be accounted for in term of relative stabilities of alkyl radical $\left(3^{0}>2^{0}>1^{0}>\right.$ methyl). The greater regioselectivity of bromination (1600:80:1) over chlorination (5:4:1) can be explained by Hammond's Postulates.

\subsection{6: Hammond's Postulates}

According to Hammond's postulates the structure of the transition state

- For an exothermic step looks more like the reactants of that step than the products.

- For an endothermic step looks more like the products of that step than the reactants. This postulate applies equally well to the transition state for a one-step reaction and to each transition state in a multi-step reaction. 


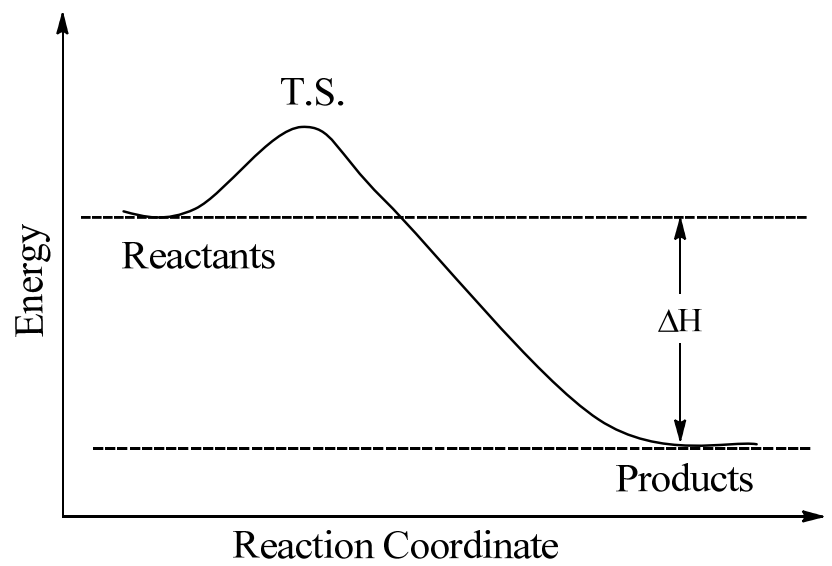

(a) Highly Exothermic Reaction

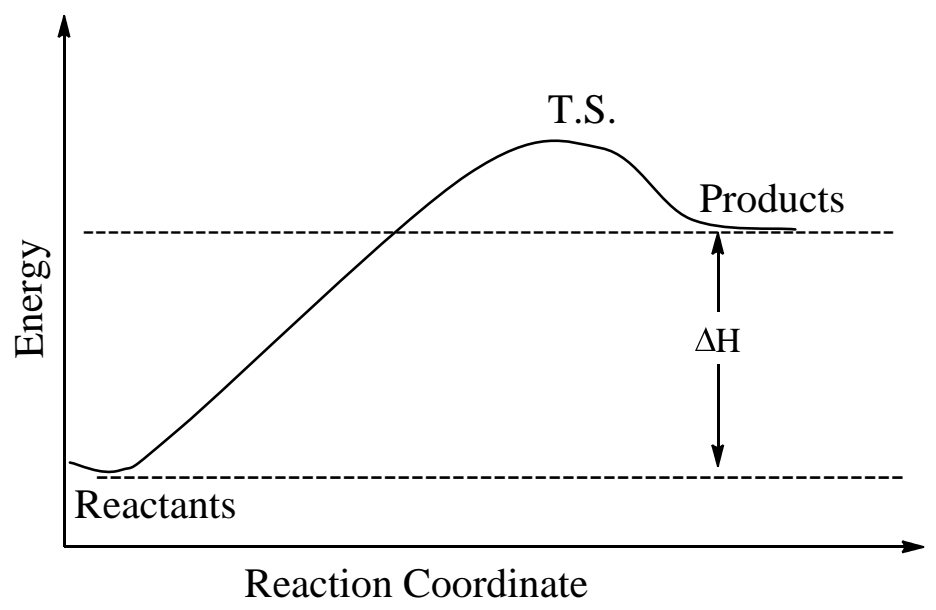

(b) Highly Endothermic Reaction

In halogenation of alkenes, hydrogen abstraction (the rate-determining step) is exothermic for chlorination but endothermic for bromination. 
Hydrogen Abstraction Steps in Chlorination

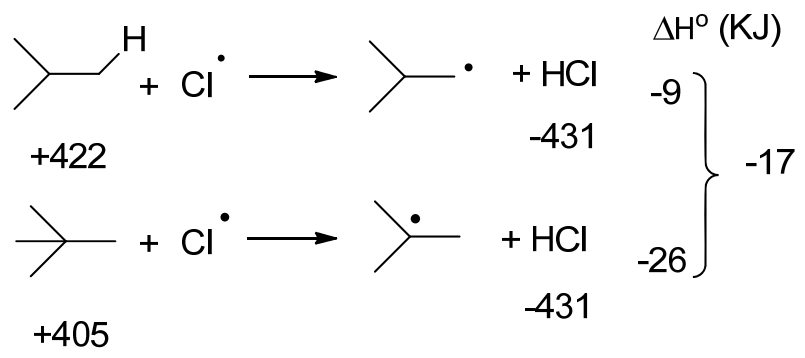

Because hydrogen abstraction for chlorination is exothermic:

- the transition state resembles the alkane and a chlorine atom

- there is little radical character on carbon in the transition state

- regioselectivity is only slightly influenced by radical stability

i. Energy diagram of primary and tertiary hydrogen abstraction by chlorine radical

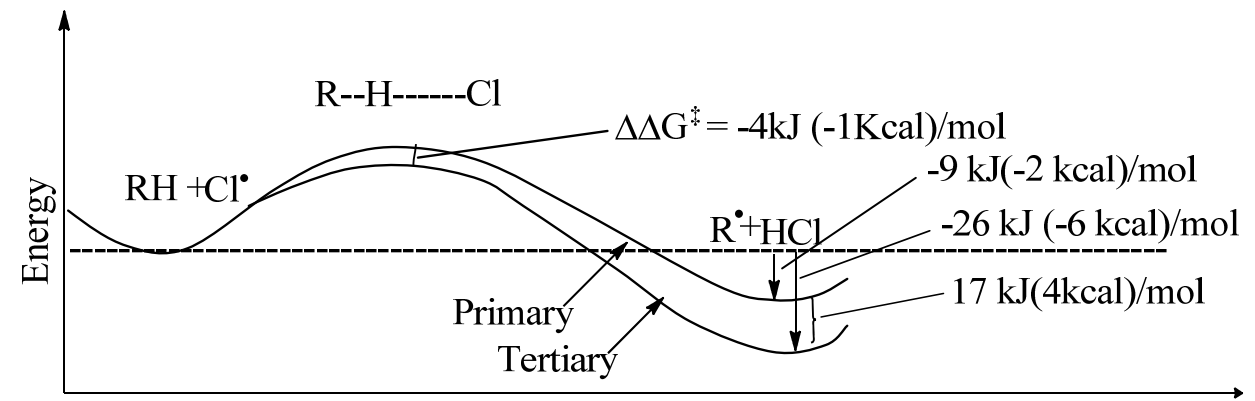

a. Chlorination

Hydrogen Abstraction Steps in Bromination

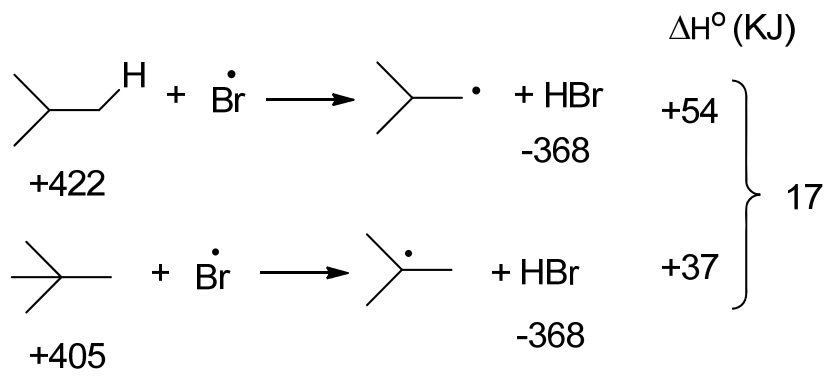


Because hydrogen abstraction for bromination is endothermic:

- the transition state resembles an alkyl radical and $\mathrm{HBr}$

- there is significant radical character on carbon in the transition state

- regioselectivity is greatly influenced by radical stability

- radical stability is $3^{\circ}>2^{\circ}>1^{\circ}>$ methyl, and regioselectivity is in the same order

ii. Energy diagram of primary and tertiary hydrogen abstraction by bromine radical

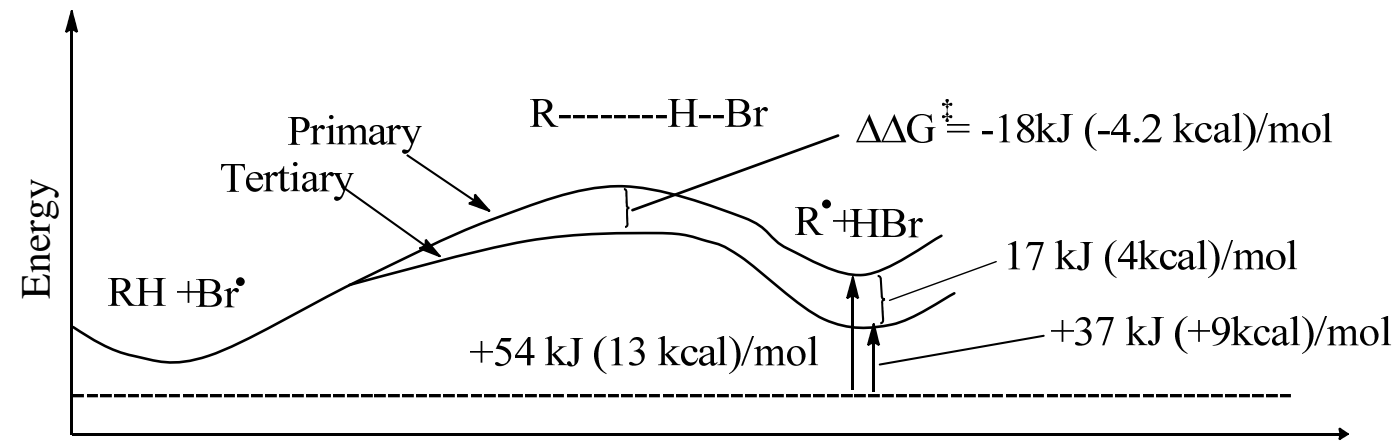

\section{b. Bromination}

\subsection{7: Reported Reactions of Picoline}

Bromination of picoline and other aromatic compounds with $\mathrm{Br}_{2} / \mathrm{SiO}_{2}$ was reported after Smith and Bye ${ }^{101}$ have reported the use of $\mathrm{NBS} / \mathrm{SiO}_{2}$ for aromatic bromination. They report that silica gel has a profound effect on the rate and the mechanism of bromination of aromatic compounds. The advantage of using $\mathrm{Br}_{2} / \mathrm{SiO}_{2}$ bromination comparing to other methods is that simple reaction condition, especially lower temperature and shorter reaction time were reported. Bromination of 2-methyl pyridine and 3-methylpyridine was carried out in 1:1 stoichiometry for 24 hours. The bromination at the methyl branch to give 2-Bromomethylpyridine and 3methylpyridine was reported. However the yield is low. $(<5)$

3-bromomethylpyridine is a known compound. It was prepared from 3aminomethylpyridine.

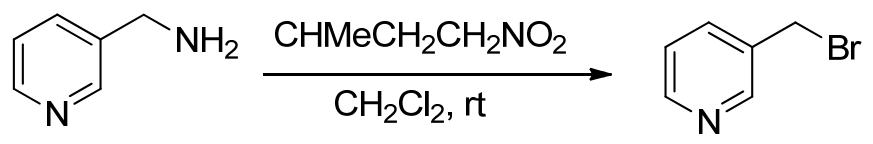


2-bromomethyl pyridine is a known compound. It is prepared either directly from 2-picoline by NBS bromination or from pyridin-2-ylmethanol.

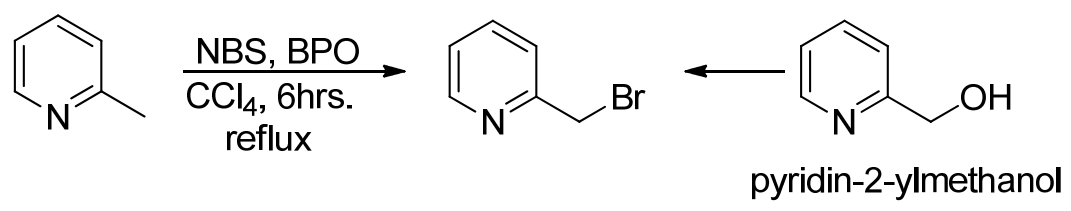

The classical approach to predict the substituent effect on the reactivity of the free radical bromination of picolines by NBS is not adequate. Hence, we revisited in this thesis the reactions to investigate the reactivities of 2, 3 and 4-picolines towards the free radical bromination by NBS and extended the work to symmetrical and unsymmetrical lutidines as well.

\subsection{8: Reported Reactions of Lutidine}

Many examples of side-chain free-radical halogenation of $N$-heterocyles have been reported in literature. ${ }^{24,25,102,103}$ but no bis(dibromomethyl) derivatives have been isolated. The preparation of tetrabromides compounds of $N$-heterocyles by photobromination of the appropriate dimethyl compound with $\mathrm{N}$-bromosuccinimide in $\mathrm{CCl}_{4}$ have been reported in literature. They report that photobromination of the 2, 3-dimethylpyridine and 3,4dimethylpyridine with $\mathrm{N}$-bromosuccinimide in $\mathrm{CCl}_{4}$ was carried out to obtained tetrabromo substituted products.

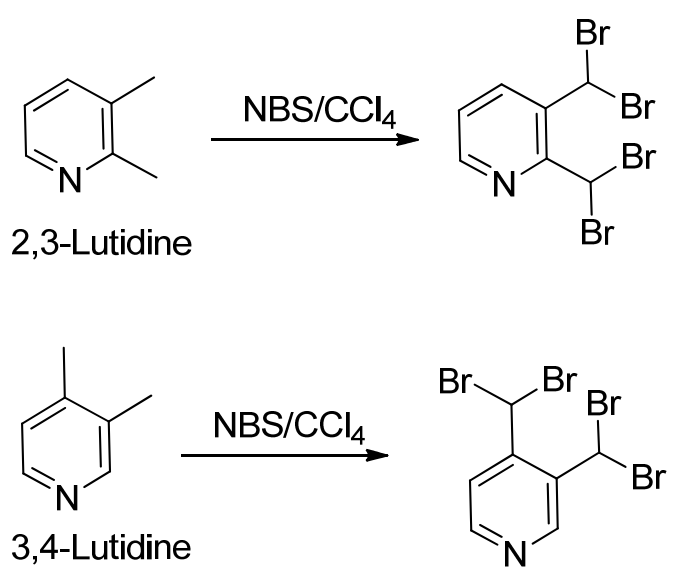


The major product by the radical bromination reaction of 2,3-dimethyl pyridine with NBS obtained was 3-(bromomethyl)-2-pyridine reported in the literature. Thus obtained monobrominated product (70) was precursor for the preparation of regioisomeric sulfide (71).

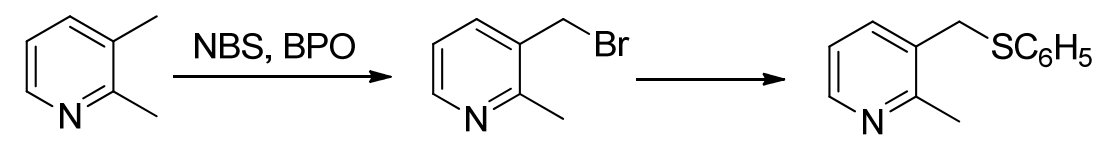

2,3-Lutidine

Preferential bromination of the C-3 methyl group of 2, 3-lutidine was unexpected because 2-picoline was found to be much more reactive than 3-picoline towards NBS bromination. ${ }^{80}$ By independent synthesis of 3-(bromomethyl)-2-methylpyridine from ethyl-2-methyl-3nicotinate (72), the location of bromine in (70) was established. First (72) was reduced to alcohol and then further converted to the bromide. ${ }^{84}$<smiles>CCOC(=O)c1cccnc1C</smiles>

A new method for side chain bromination of aromatic and heteroaromatic methyl groups by microwave-assisted solid phase NBS without radical initiator was developed. 2(bromomethyl)-6-pyridine was synthesized from 2, 6- Lutidine by one step solid phase Nbromosuccinimide reaction without radical initiator by Microwave irradiation. ${ }^{104}$ In case of 2, 6 Lutidine only side chain bromo substituted product was reported, which may be due to less electron density in the ring compared with other examples given in the paper.

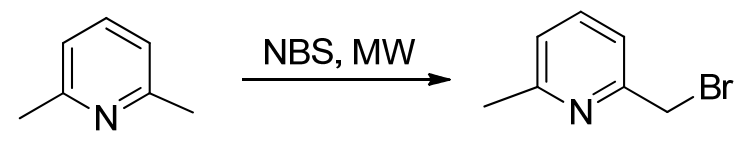

2,6-Lutidine 2-(bromomethyl)-6-methylpyridine 
The data also suggest that the electron density in all the methyl substituted starting materials having simple aromatic and heterocyclic ring like pyridine, pyrizine and phenanthroline, is less compared to pyrizine amides and thus prevents ring bromination but mostly favors side chain bromination. Several attempts were made to convert the methyl groups of 2, 6-Lutidine substituent in the process of the synthesis of nonane ring system. ${ }^{24}$ The reaction of 2, 6-Lutidine and NBS gave some mono bromo substituted 2-(bromomethyl)-6-methylpyridine. Small amount of 2, 6-bis (bromomethyl) pyridine and large quantity of acid soluble polymer products obtained by the reaction of dilithium salt of 2, 6-lutidine and bromine in ether was reported.

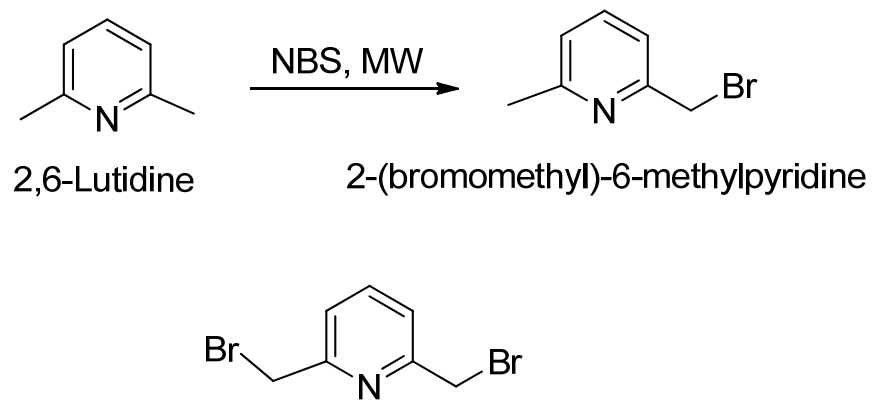

2,6-bis(bromomethyl)pyridine

\subsection{9: Research Aim}

NBS is a highly selective free radical brominating agent. The main goal of research work is to determine the reactive site and products of unsymmetrical dimethylpyridine and to achieve better understanding of the regioselectivity of the compound towards free radical NBS bromination (10\%) and with stoichiometric amounts. NBS bromination of the unsymmetrical dimethyl pyridine was carried out with limiting NBS. Mono brominated product was initially obtained by using $10 \%$ of the reagent to find the reactive site of the compound. Reaction is further carried out in1:1 equivalent to obtain a sufficient amount of monobrominated product so that it can be isolated and analyzed with the help of GC/MS ${ }^{1} \mathrm{H}$ NMR and NOE. Namely 2,3-, 2,5-, 2,4- and 3,4 dimethyl pyridine were reacted with NBS with standard methods mention in the experimental section and the data were obtained and discussed. Our research focus is to accomplish the understanding of regioselectivity towards NBS bromintion of unsymmetrical dimethyl pyridine so no effort was done to isolate and analyze the polybrominated products. 


\section{CHAPTER 2: Experimental Result and Discussion}

\section{1: General Reaction Protocol}

Reactions were carried in the Radley Carousel Reactor using 50 ml reactor tubes. Temperature was controlled by a thermister. Reactions products were analyzed by GC/MS (Varian Saturn 2000) using a 30mx 0.25mmx 0.25 $\mu \mathrm{m}$ UF-35ms column at $250^{\circ} \mathrm{C}$ and 81.7 $\mathrm{ml} / \mathrm{min}$ and $300 \mathrm{MHz}$ or $500 \mathrm{MHz}{ }^{1} \mathrm{H} \mathrm{NMR}$ spectrometer (Bruker Avance) in $\mathrm{CDCl}_{3}$. Purification was carried out by preparative TLC (silica gel 30gm plus 60gm distilled water) and a purified product characterized by GC/MS and ${ }^{1} \mathrm{H}$ NMR.

\section{2: GC/MS analysis:}

In analyzing the reactions with NBS as the limiting reagent (see below), chromatogram showed a large peak for recovered starting material. Smaller peaks at a longer retention time were observed. No attempts were made to purify the crude reaction mixture so several byproducts (benzoic acid, etc) could be observed but reaction products could be selected by displaying chromatograms of the critical mass numbers. In particular all products showed a molecular ion, with a parent ion for loss of bromine.

To obtain samples for H NMR analysis, the reaction was carried out with stoichiometric amounts of NBS. Such reaction conditions produced larger amounts of polybrominated materials, but chromatography permitted isolation of those materials obtained from the limiting case, no attempt was made to isolate the 'new' products. The chemical shift of methyl groups in the 2-position ( 2.55) is substantially downfield form 3 and 4-positions ( 2.3). Normally this permitted directed assignment of the product structure.

\section{3: Standard Procedure}

The reactor tube was charged with $0.84 \mathrm{gm}$ (9 mmol) of 2-methyl pyridine (F.W. 93.13), 0.16gm (0.9 mmol, $10 \%$ n-bromosuccinamide (NBS, F.W. 178) and 10mg benzoyl peroxide. $10 \mathrm{ml}$ trifluorotoluene solvent was added in the reaction mixture. The reaction mixture tube is placed in the carousel reactor and heated with stirring to $85^{\circ} \mathrm{C}$ for 8 hours. 
The red reaction mixture was cooled and filtered away the solid remaining. $100 \mathrm{ml}$ of diethyl ether was added to the filtrate. Thus obtained mixture was washed with $50 \mathrm{ml}$ of bicarbonate solution and $50 \mathrm{ml}$ of sodium sulphite solution with the help of separating funnel. After washing, the organic layer was dried by adding anhydrous magnesium sulfate. The solid magnesium sulfate was filtered away and thus obtained anhydrous organic layer was evaporated to obtained concentrated mixture of the products. The products were analyzed with the help of GC/MS having a $30 \mathrm{~m} \times 0.25 \mathrm{~mm} \times 0.25 \mu \mathrm{m}$ UF- $35 \mathrm{~ms}$ column at $250^{\circ} \mathrm{C}$ and $81.7 \mathrm{ml} / \mathrm{min}$ and ${ }^{1} \mathrm{H}$ NMR. The injection temperature $250^{\circ} \mathrm{C}$, split ratio 100:1 with constant pressure of 5 psi. The column temperature rises from $50^{\circ} \mathrm{C}$ to $250{ }^{\circ} \mathrm{C}$ at the rate of $10^{\circ} \mathrm{C} / \mathrm{min}$. with 3 minutes hold at the beginning and 6 minutes hold at the end. The mass spectrum using electron impact ionization technique with 20-200 mass unit.

The reaction was carried with 1:0.1 (10\%) substrate and NBS first and then 1:1 stoichiometry to obtain the substantial amount of brominated product for analysis. Brominated product of interest was isolated with the help of preparative TLC/ Flash Chromatography. Different solvent composition EtOAc or $\mathrm{CH}_{2} \mathrm{Cl}_{2}$ and petroleum ether ware used as mobile phase for TLC. Finally brominated product was confirmed by ${ }^{1} \mathrm{H}$ NMR.

\subsubsection{Bromination of 2-Methylpyridine}

The bromination of 2-picoline was carried out according to the standard protocol.<smiles>Cc1ccccn1</smiles>

2-picoline

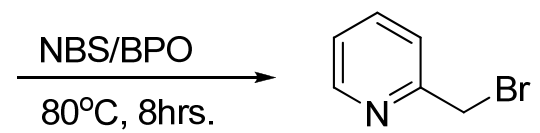

2-(bromomethyl)pyridine

Mono-brominated product was isolated by preparative TLC. Mixture of 75\% dichloromethane and $25 \%$ petroleum ether was used as eluent for TLC. NMR sample was made $\mathrm{CDCl}_{3}$. The ${ }^{1}$ HNMR confirmed that the mono-bromination occurred at methyl branch to give 2(bromomethyl) pyridine. The spectral data were identical to those reported in the literature. ${ }^{105}$

\subsubsection{Bromination of 3-Methylpyridine}

Bromination of 3-Picoline was carried out according to the standard procedure. The products were analyzed with the help of GC/MS having a 30m x $0.25 \mathrm{~mm} \times 0.25 \mu \mathrm{m}$ UF-35ms 
column at $250^{\circ} \mathrm{C}$ and $81.7 \mathrm{ml} / \mathrm{min}$ revealed two peaks corresponding to remaining starting material and a monobrominated product. The brominated product was observed at retention time 20.14 minutes.

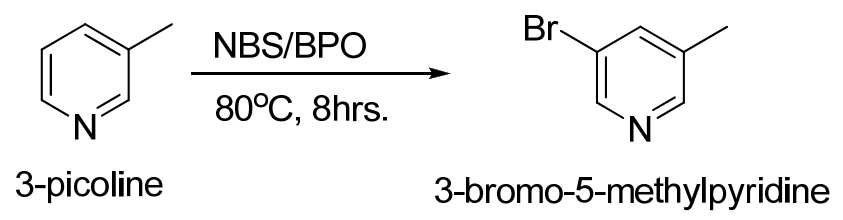

The monobrominated product was isolated by preparative TLC, using a mixture of 25\% dichloromethane and 75\% petroleum ether as eluent for TLC. The NMR sample was prepared in $\mathrm{CDCl}_{3}$. The ${ }^{1} \mathrm{H}$ NMR confirmed that the mono-bromination did not occur at the methyl branch but in the ring to give 3-bromo-5-methylpyridine. The spectral data were identical to those reported in the literature. ${ }^{106}$

\subsubsection{Bromination of 4-Methylpyridine}

Bromination of 4-Picoline was carried out according to the standard procedure. The product mixture was analyzed with the help of GC/MS having a 30m x $0.25 \mathrm{~mm} \times 0.25 \mu \mathrm{m}$ UF$35 \mathrm{~ms}$ column at $250{ }^{\circ} \mathrm{C}$ and $81.7 \mathrm{ml} / \mathrm{min}$ revealed two peaks corresponding to monobrominated and dibrominated products at retention time of 15.03 and 18.37 minutes in a ratio of 1:9.5.

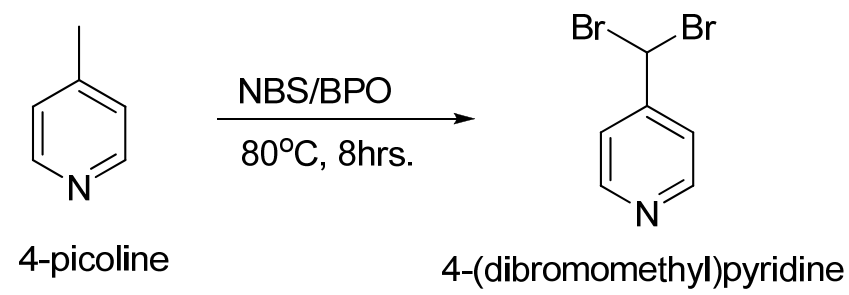

Monobrominated product was not isolated but dibrominated product was isolated by preparative TLC, using mixture of 75\% dichloromethane and 25\% petroleum ether as eluent. The NMR sample was prepared in $\mathrm{CDCl}_{3}$. The ${ }^{1} \mathrm{H}$ NMR confirmed that the dibromination occurred at methyl branch to give 4- (dibromomethyl) pyridine. The spectral data were identical to those reported in literature. ${ }^{107}$ 


\subsubsection{Bromination of 2, 4-dimethylpyridine}

The bromination of 2,4-dimethylpyridine was carried out according to the standard procedure. The GC/MS analysis was carried out using a $30 \mathrm{~m}$ x $0.25 \mathrm{~mm}$ x $0.25 \mu \mathrm{m}$ UF-35ms column at $250^{\circ} \mathrm{C}$ and $81.7 \mathrm{ml} / \mathrm{min}$.

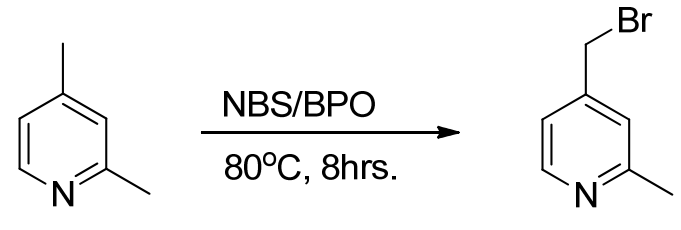

$$
\text { 2,4-dimethylpyridine }
$$

Monobrominated as well as dibrominated products was obtained at retention time of 15.80 and 18.97 minutes in a ratio of 1:1.36 was obtained including several other side products. Monobrominated product was not isolated but dibrominated product was isolated by preparative TLC using 3:1 mixture of $\mathrm{CH}_{2} \mathrm{Cl}_{2}$ and petroleum ether as eluent. The NMR sample was prepared in $\mathrm{CDCl}_{3}$. The $\mathrm{H}$ NMR confirmed that the dibrominated product is 4, 4'-(dibromomethyl) pyridine. Hence, it concludes that the monobrominated product is (73, p-47). ${ }^{1} \mathrm{H} \mathrm{NMR}\left(\mathrm{CDCl}_{3}\right.$, $500 \mathrm{MHz}) \delta 2.45$ (s, 3H), 4.64 (s, 2H), 7.50 (m, 2H), 8.13 (s, 1H)

\subsubsection{Bromination of 2, 5-dimethylpyridine}

The bromination of 2,5- lutidine was carried out according to the standard procedure. GC/MS analysis using a 30m x $0.25 \mathrm{~mm} \times 0.25 \mu \mathrm{m}$ UF-35ms column at $250^{\circ} \mathrm{C}$ and $81.7 \mathrm{ml} / \mathrm{min}$ showed four peaks including starting material, mono (74) and dibrominated (75) products. The retention time observed for mono and dibrominated products are $15.91 \mathrm{~min}$. and $19 \mathrm{~min}$.

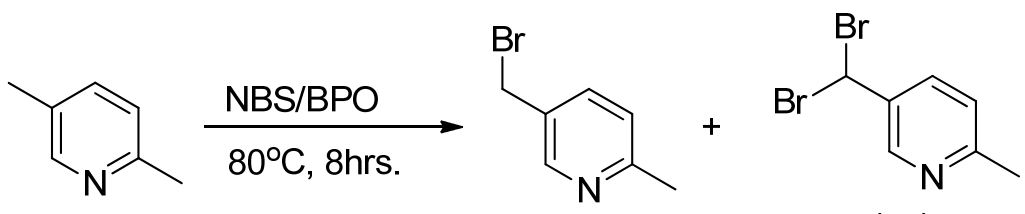

2,5-dimethylpyridine

(74) 
Monobrominated product was isolated by preparative TLC using mixture of $20 \%$ dichloromethane and $80 \%$ petroleum ether. NMR sample was prepared in $\mathrm{CDCl}_{3}$. The NMR data concludes the monobrominated product is 5-(dibromomethyl)-2-methylpyridine (74, p-48). ${ }^{1} \mathrm{H}$ NMR ( $\left.\mathrm{CDCl}_{3}, 500 \mathrm{MHz}\right) \delta 2.40$ (s, 3H), 4.61 (s, 1H), 7.1 (s, 1H), 7.49 (s, 1H), 8.44 (s, 1H). Mass spectrum (74, p-54); m/z 185 ( $\left.\mathrm{M}^{+}, 15 \%\right), 106$ (100\%), 77(47\%)

\subsubsection{Bromination of 3, 4-dimethylpyridine}

The bromination of 3, 4- lutidine was carried out according to the standard procedure. GC/MS analysis using a $30 \mathrm{~m} \times 0.25 \mathrm{~mm} \times 0.25 \mu \mathrm{m}$ UF- $35 \mathrm{~ms}$ column at $250^{\circ} \mathrm{C}$ and $81.7 \mathrm{ml} / \mathrm{min}$ showed two peaks one for starting material and one for the dibrominated product (76) with retention time $19.63 \mathrm{~min}$.

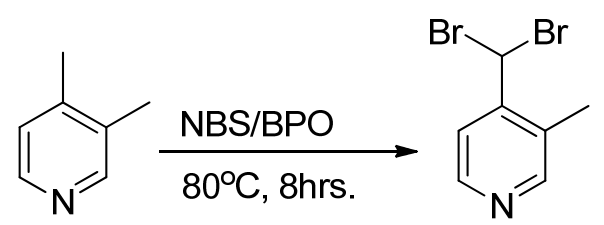

3,4-dimethylpyridine

(76)

Dibrominated product was isolated by Thin Layer Chromatography using a mixture of $40 \%$ EtOAc and $60 \%$ Petroleum ether as eluent. $\mathrm{CDCl}_{3}$ was used to prepare the NMR sample. The HNMR as well as NOE data concludes the brominated product is 4-(bromomethyl)-3-methyl pyridine (76, p-49). ${ }^{1} \mathrm{H}$ NMR ( $\left.\mathrm{CDCl}_{3}, 500 \mathrm{MHz}\right) \delta 2.44$ (s, 3H), 6.76 (s, 1H), 7.69 (d, $J=5,1 \mathrm{H}$ ), 8.43 (d, 1H), 8.57 (d, J=5, 1H); mass spectrum (p-55); m/z 265 (M+1, 8\%), 184 (64\%), 105 (100\%), 78(29\%). NOE data analysis concludes the dibromo is in 4-methyl group not at 3methyl group. Irradiation of the signal results in enhancement of the signals at 6.76 and 8.43.

\subsubsection{Bromination of 2, 3-dimethylpyridine}

The bromination of 2, 3-picoline was carried out according to the standard procedure. The products were analyzed with the help of GC/MS. Retention time for monobrominated product is $15.88 \mathrm{~min}$. 


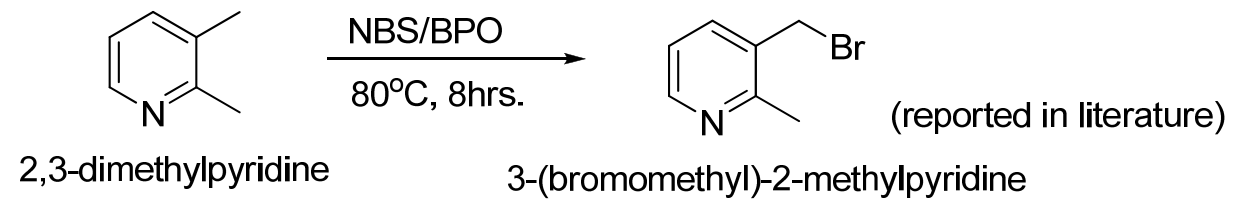

Mono-brominated product was isolated by preparative TLC using a mixture of 75\% $\mathrm{CH}_{2} \mathrm{Cl}_{2}$ and $25 \%$ petroleum ether as eluent. The NMR sample was prepared in $\mathrm{CDCl}_{3}$. The $\mathrm{H}-$ NMR confirmed that the monobromination occurred at methyl branch to give 3-(bromomethyl) pyridine. The spectral data were identical to those reported in literature. ${ }^{108}$ mass spectrum (p-52) m/z 185 (M+, 23\%), 106 (100\%), 79(51\%).

\section{Result and Discussion:}

Bromination at the farthest methyl group from nitrogen was obtained during selective free radical bromination of unsymmetrical dimethyl pyridines. The free radical bromination of 2,3-, 2,4- and 2,5-dimethyl pyridine by NBS produce 3-(bromomethyl)-2-methylpyridine, 4(bromomethyl)-2-methylpyridne and 5-(bromomethyl)-2-methyl pyridine as shown in the Table respectively.

Table 5: Reactants and products of NBS bromination

\begin{tabular}{|l|l|}
\hline Reactant & \multicolumn{1}{|c|}{ Remarks } \\
\hline literature
\end{tabular}


In case of 3,4-dimethyl pyridine monobrominated product was not obtained even when only $10 \%$ NBS was used. It is surprising that a monobrominated product was formed in case of 2,3- and 2,4-dimethyl pyridine but monobromination does not takes place in case of 3,4methylpyridine . 3,4-dimethylpyridine produce 4-(dibromomethyl)-3-methylpyridine as shown in the table (entry 78). This may be because 4-methyl group is most reactive site as it has highest electron density comparing 2- and 3-methyl group as it lies farthest from the inductively deactivating $\mathrm{N}$ in the pyridine ring.

The monobromination causes the $\mathrm{C}-\mathrm{H}$ bond to be more polar and highly reactive so that dibromination takes place so rapidly that no monobrominated product remains in case of 3,4 dimethylpyridne. The case for 2,4- dimethylpyridine is similar, but in this case monobrominated product can be observed. The methyl group is an electron releasing group. The methyl group in 3- position is in closer proximity in 3,4-dimethylpyridine than 2-methyl group in 2,4-dimethylpyridine this may be the cause that the electron density in 4-methyl in 3,4-dimethylpyridine is higher comparing 4-methyl group in 2,4-dimethylpyridine.

We also reran some of the reactions of monomethylpyridine from the literature to get better understanding to free radical NBS bromination. The product obtained from the free radical NBS bromination of 2-, 3- and 4-methylpyridine are given as follows in the table 6.

Table 6: Reactant and Products of monomethylpyridine

\begin{tabular}{|l|l|}
\hline Reactant & Product \\
\hline
\end{tabular}


Free radical bromination of 4-methylpyridine gives only dibrominated product that can be explained as in case of 3,4-dimethylpyridine as above. In the case of 3-methylpyridine bromination at methyl group does not takes place instead ring substituted product was obtain as shown in the table 6 (entry 79) probably by non radical mechanism. It is not clear why 3-methyl group in 3-methylpyridine is not brominated. In the case of 2-bromomethylpyridine our result is consistently low yield in keeping with the literature.

\section{Conclusion:}

The competition between 2, 3-, 2, 4- and 2, 5-dimethyl pyridine towards bromination results with bromination in the methyl group farthest from the $\mathrm{N}$ in the ring. The results are consistent with theory that the electronegative attacking radicals prefer positions of high electron density and abstract hydrogens from carbon atoms, which are farthest from the electronwithdrawing substituents. Regioselectivity of bromination in unsymmetrical dimethyl Pyridine shows that Nitrogen in ring is deactivating inductively.

\section{Future Work}

We want to carry out the study relative rates by competitive free radical bromination reaction between different dimethylpyridines. Free radical bromination of mixtures of 1- and 4-; 3- and 2, 3-; 2- and 2,6 etc by NBS and observing the competitive bromination between mixtures of two or more different picolines or lutidines. This result will provide the relative rate of the free radical bromination of methyl branch at different position as well as in different compounds. We would like to extend this work with polymethyl compound; collidines. 
${ }^{1} \mathrm{H}$ NMR of 4-(bromomethyl)-2-methylpyridne (73, p-42)

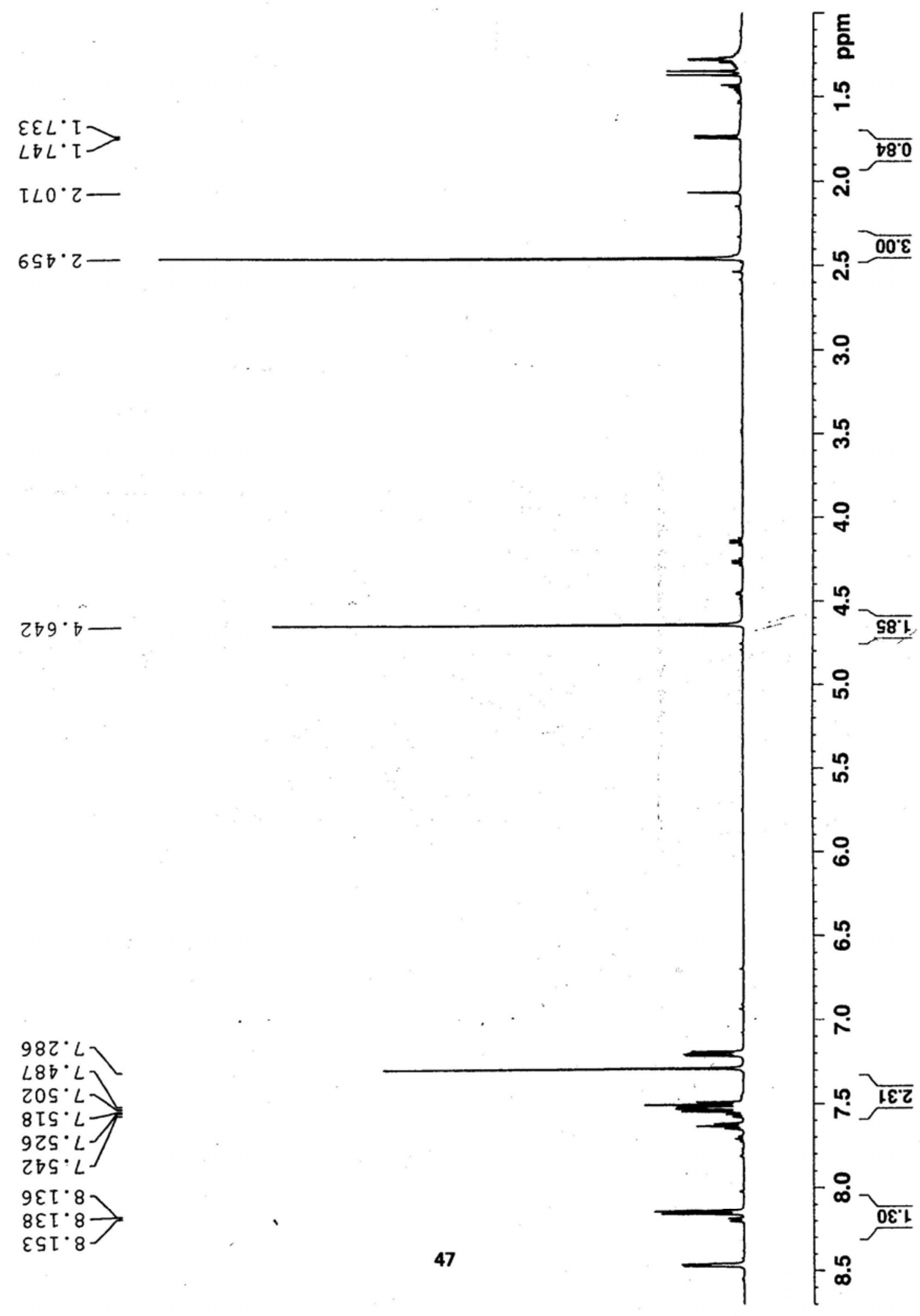




\section{${ }^{1} \mathrm{H}$ NMR of 5-bromomethyl-2-methylpyridne (74, p-42)}

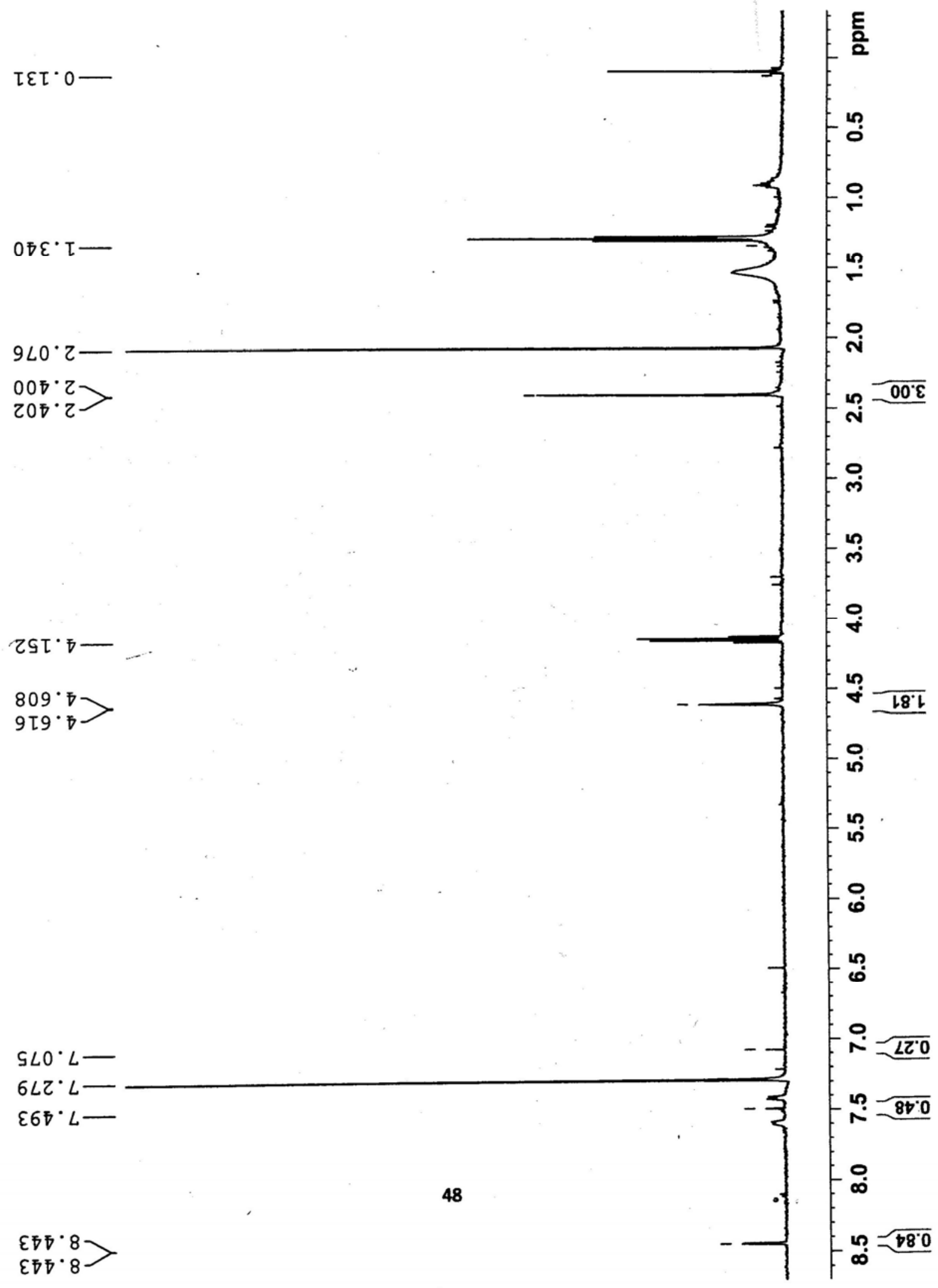


${ }^{1} \mathrm{H}$ NMR of 4-(dibromomethyl)-3-methylpyridne (76, p-43)
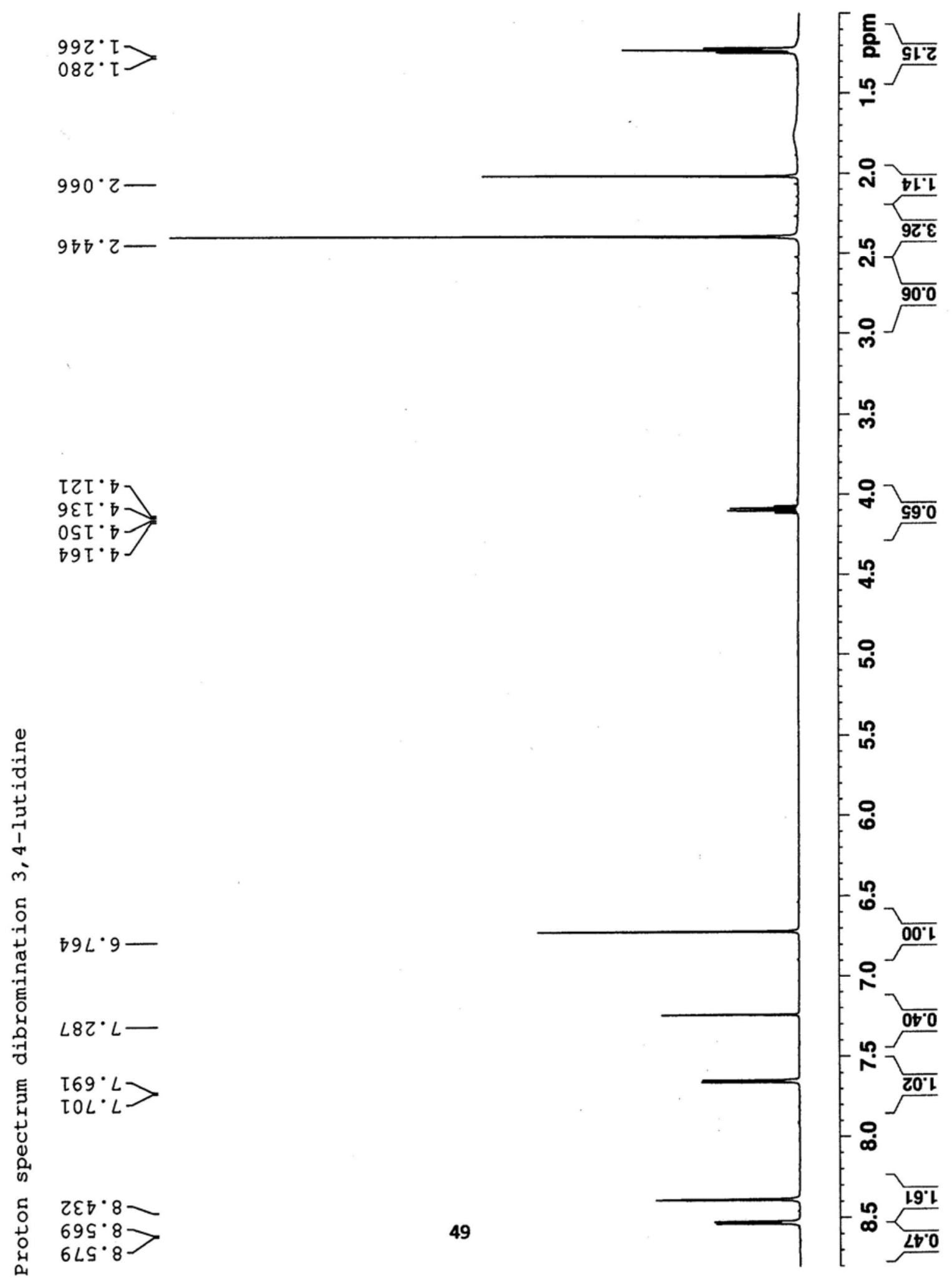
NOE of 4-(dibromomethyl)-3-methylpyridne irradiated at 6.75

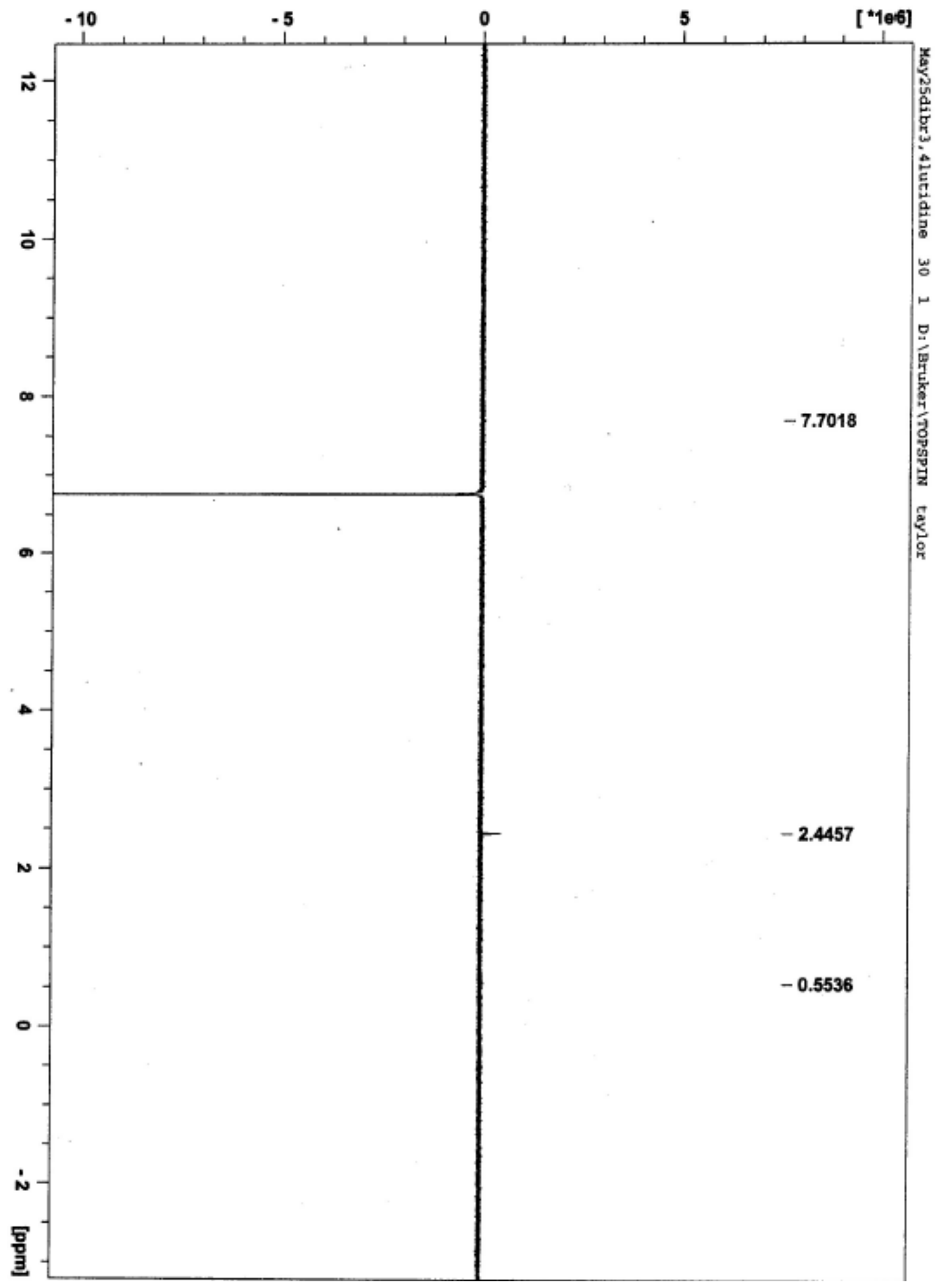


NOE of 4-(dibromomethyl)-3-methylpyridne irradiated at 2.43

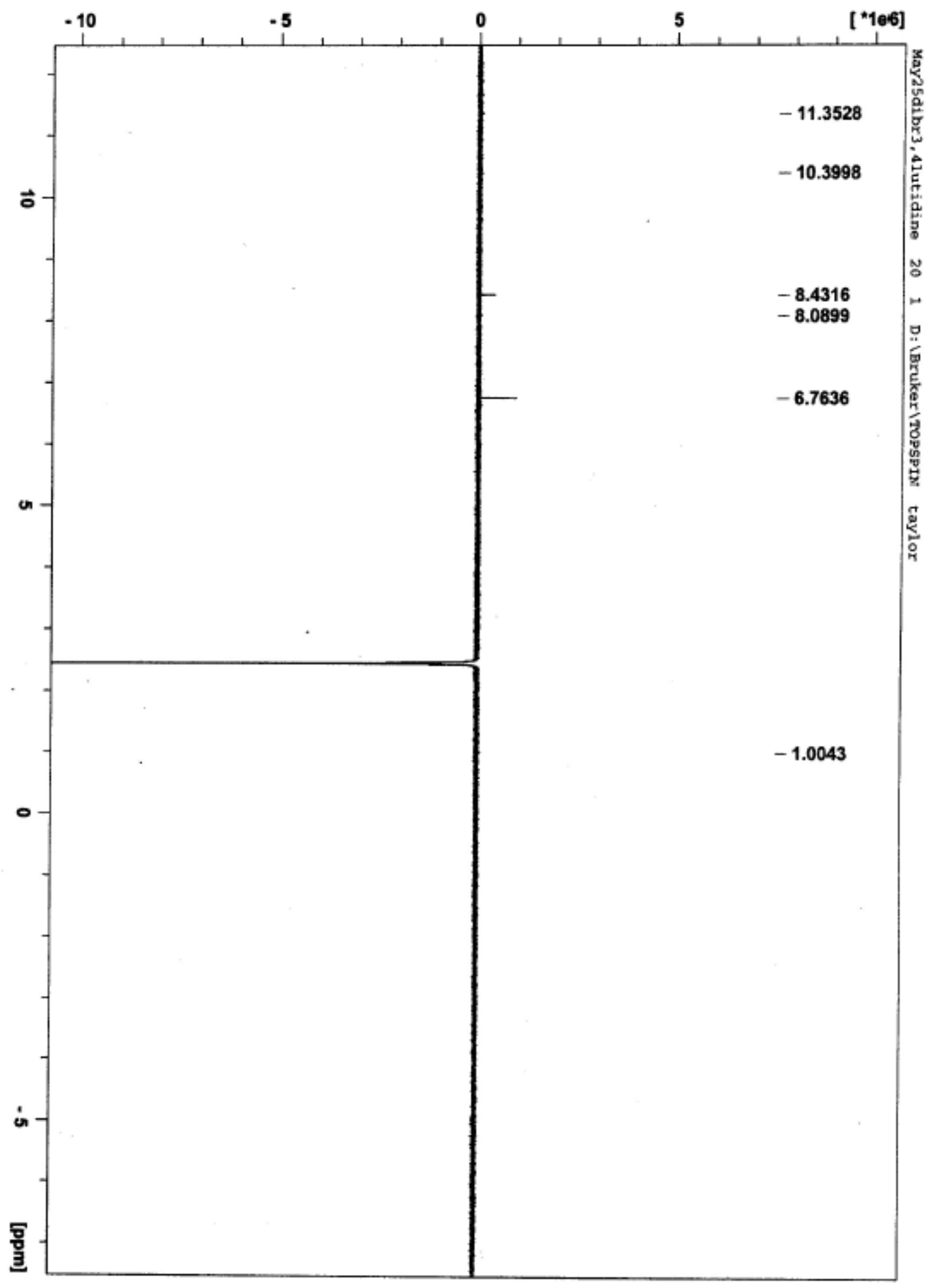




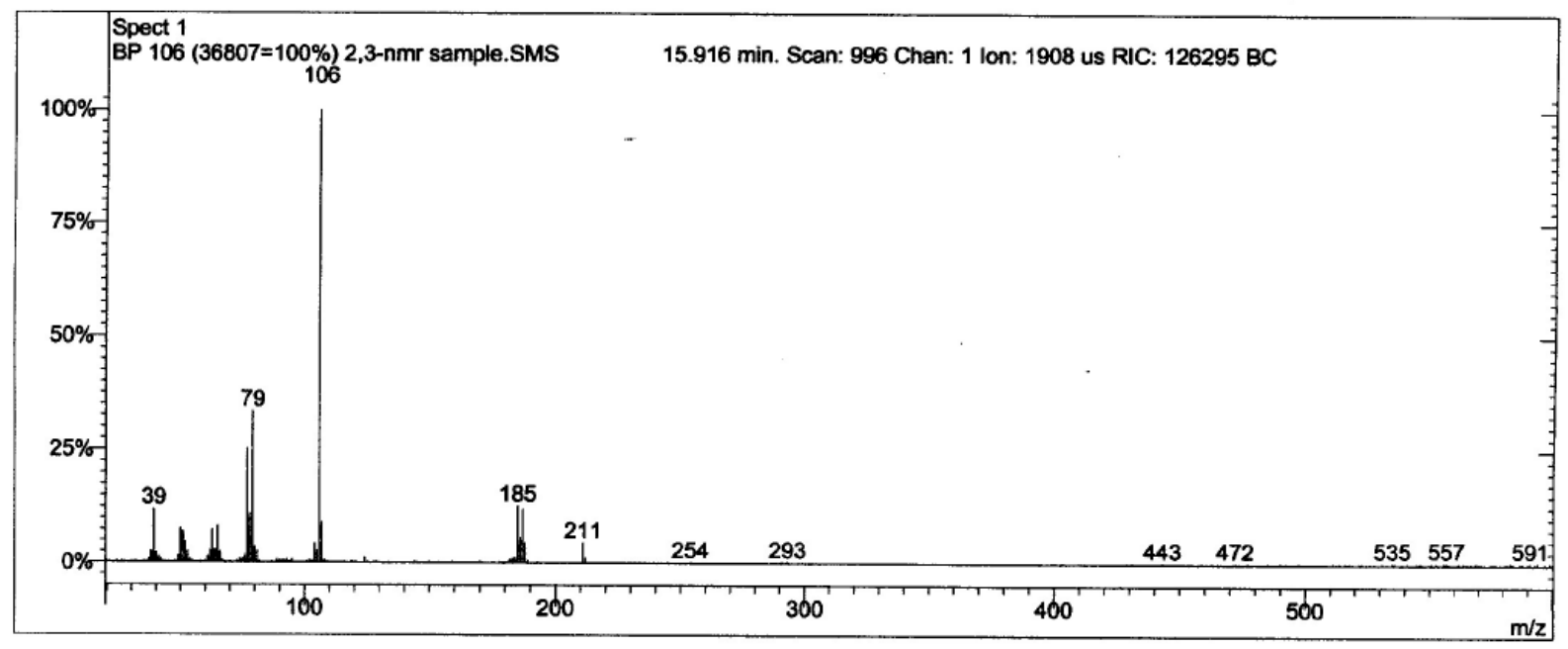

Spectrum from c:1... Ipyridine unsymml2,312,3-nmr sample.sms

Scan No: 996, Time: 15.916 minutes

No averaging. Background corrected.

Comment: 15.916 min. Scan: 996 Chan: 1 Ion: 1908 us RIC: 126295 BC

Pair Count: 155 MW: 0 Formula: None CAS No: None Acquired Range: 20 - 600

\begin{tabular}{|c|c|c|c|c|c|c|c|c|c|c|c|}
\hline Ion & Int & $8 \mathrm{BP}$ & Ion & Int & zBP & Ion & Int & $8 \mathrm{BP}$ & Ion & Int & $8 \mathrm{BP}$ \\
\hline 20 & 91 & 0 & 74 & 313 & 1 & 178 & 104 & 0 & 533 & 69 & 0 \\
\hline 21 & 87 & 0 & 75 & 313 & 1 & 181 & 118 & 0 & 535 & 164 & 0 \\
\hline 24 & 106 & 0 & 76 & 644 & 2 & 182 & 288 & 1 & 536 & 157 & 0 \\
\hline 26 & 111 & 0 & 77 & 9196 & 25 & 183 & 347 & 1 & 539 & 96 & 0 \\
\hline 27 & 78 & 0 & 78 & 3970 & 11 & 184 & 445 & 1 & 541 & 112 & 0 \\
\hline 30 & 62 & 0 & 79 & 12254 & 33 & 185 & 4646 & 13 & 545 & 110 & 0 \\
\hline $31^{\circ}$ & 91 & 0 & 80 & 1275 & 3 & 186 & 2017 & 5 & 546 & 112 & 0 \\
\hline 32 & 103 & 0 & 81 & 959 & 3 & 187 & 4410 & 12 & 547 & 113 & 0 \\
\hline 35 & 104 & 0 & 82 & 117 & 0 & 188 & 1610 & 4 & 550 & 100 & 0 \\
\hline 36 & 104 & 0 & 87 & 104 & 0 & 189 & 193 & 1 & 551 & 139 & 0 \\
\hline 37 & 321 & 1 & 89 & 274 & 1 & 193 & 45 & 0 & 553 & 131 & 0 \\
\hline 38 & 938 & 3 & 90 & 167 & 0 & 211 & 1661 & 5 & 556 & 155 & 0 \\
\hline 39 & 4336 & 12 & 91 & 212 & 1 & 212 & 409 & 1 & 557 & 183 & 0 \\
\hline 40 & 772 & 2 & 92 & 170 & 0 & 217 & 75 & 0 & 558 & 122 & 0 \\
\hline 41 & 491 & 1 & 93 & 267 & 1 & 254 & 58 & 0 & 562 & 149 & 0 \\
\hline 42 & 304 & 1 & 94 & 104 & 0 & 291 & 86 & 0 & 564 & 165 & 0 \\
\hline 43 & 81 & 0 & 95 & 268 & 1 & 293 & 91 & 0 & 565 & 103 & 0 \\
\hline 44 & 65 & 0 & 101 & 82 & 0 & 429 & 50 & 0 & 566 & 103 & 0 \\
\hline 45 & 53 & 0 & 102 & 177 & 0 & 431 & 58 & 0 & 567 & 81 & 0 \\
\hline 47 & 44 & 0 & 103 & 159 & 0 & 443 & 91 & 0 & 569 & 181 & 0 \\
\hline 48 & 117 & 0 & 104 & 1577 & 4 & 472 & 104 & 0 & 570 & 121 & 0 \\
\hline 49 & 549 & 1 & 105 & 972 & 3 & 477 & 89 & 0 & 571 & 131 & 0 \\
\hline 50 & 2799 & 8 & 106 & 36807 & 100 & 485 & 78 & 0 & 575 & 102 & 0 \\
\hline 51 & 2572 & 7 & 107 & 3310 & 9 & 490 & 94 & 0 & 576 & 152 & 0 \\
\hline 52 & 1667 & 5 & 108 & 248 & 1 & 492 & 98 & 0 & 577 & 109 & 0 \\
\hline 53 & 908 & 2 & 109 & 80 & 0 & 497 & 126 & 0 & 581 & 105 & 0 \\
\hline 54 & 273 & 1 & 116 & 91 & 0 & 498 & 104 & 0 & 582 & 120 & 0 \\
\hline 55 & 85 & 0 & 119 & 121 & 0 & 499 & 65 & 0 & 583 & 175 & 0 \\
\hline 58 & 85 & 0 & 120 & 104 & 0 & 500 & 78 & 0 & 584 & 116 & 0 \\
\hline 60 & 90 & 0 & 124 & 441 & 1 & 511 & 131 & 0 & 587 & 136 & 0 \\
\hline 61 & 537 & 1 & 125 & 91 & 0 & 514 & 87 & 0 & 588 & 148 & 0 \\
\hline 62 & 982 & 3 & 128 & 90 & 0 & 516 & 98 & 0 & 590 & 116 & 0 \\
\hline 63 & 2733 & 7 & 129 & 83 & 0 & 518 & 104 & 0 & 591 & 183 & 0 \\
\hline 64 & 1048 & 3 & 144 & 117 & 0 & 519 & 74 & 0 & 593 & 122 & 0 \\
\hline 65 & 2997 & 8 & 145 & 79 & 0 & 522 & 85 & 0 & 594 & 169 & 0 \\
\hline 66 & 849 & 2 & 150 & 83 & 0 & 523 & 98 & 0 & 597 & 155 & 0 \\
\hline 67 & 201 & 1 & 154 & 63 & 0 & 526 & 94 & 0 & 599 & 172 & 0 \\
\hline 68 & 77 & 0 & 159 & 91 & 0 & 528 & 104 & 0 & 600 & 160 & 0 \\
\hline 73 & 106 & 0 & 170 & 117 & 0 & 531 & 138 & 0 & & & \\
\hline
\end{tabular}




\section{4-bromo-2-methylpyridine}

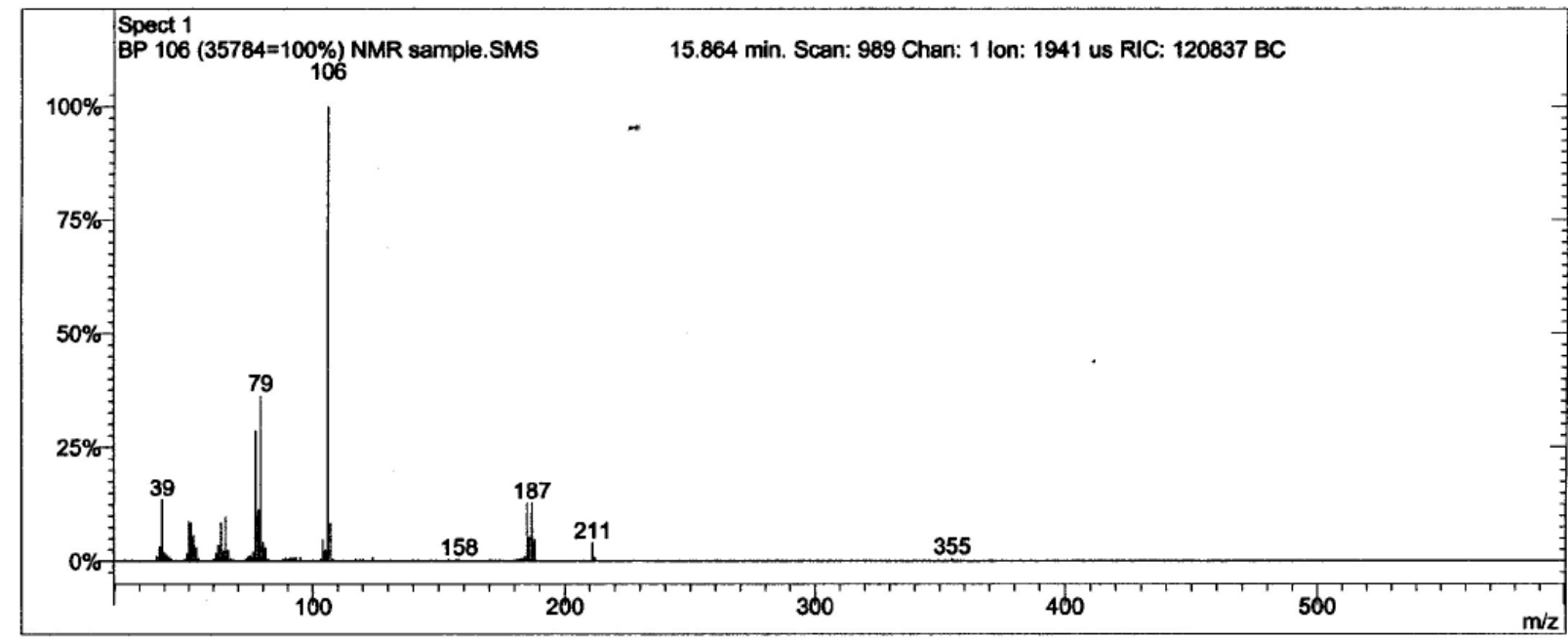

Spectrum from c:l... Ipyridine unsymml2,4lnmr sample.sms Scan No: 989, Time: 15.864 minutes

No averaging. Background corrected.

Comment: 15.864 min. Scan: 989 Chan: 1 lon: 1941 us RIC: 120837 BC

Pair Count: 78 MW: 0 Formula: None CAS No: None Acquired Range: 20 - 600

\begin{tabular}{|c|c|c|c|c|c|c|c|c|c|c|c|}
\hline Ion & Int & $\frac{8}{8 P}$ & Ion & Int & \&BP & Ion & Int & 을 & Ion & Int & $8 \mathrm{BP}$ \\
\hline 24 & 77 & 0 & 63 & 3009 & 8 & 92 & 231 & 1 & 149 & 74 & 0 \\
\hline 27 & 77 & 0 & 64 & 798 & 2 & 93 & 320 & 1 & 154 & 77 & 0 \\
\hline 37 & 412 & 1 & 65 & 3508 & 10 & 95 & 309 & 1 & 157 & 77 & 0 \\
\hline 38 & 1096 & 3 & 66 & 834 & 2 & 100 & 64 & 0 & 158 & 115 & 0 \\
\hline 39 & 4833 & 14 & 67 & 188 & 1 & 103 & 138 & 0 & 170 & 77 & 0 \\
\hline 40 & 735 & 2 & 68 & 90 & 0 & 104 & 1683 & 5 & 171 & 103 & 0 \\
\hline 41 & 479 & 1 & 73 & 124 & 0 & 105 & 843 & 2 & 174 & 77 & 0 \\
\hline 42 & 373 & 1 & 74 & 396 & 1 & 106 & 35784 & 100 & 180 & 77 & 0 \\
\hline 43 & 115 & 0 & 75 & 447 & 1 & 107 & 2994 & 8 & 181 & 90 & 0 \\
\hline 45 & 50 & 0 & 76 & 699 & 2 & 108 & 141 & 0 & 182 & 154 & 0 \\
\hline 48 & 90 & 0 & 77 & 10225 & 29 & 112 & 64 & 0 & 183 & 218 & 1 \\
\hline 49 & 604 & 2 & 78 & 4050 & 11 & 117 & 103 & 0 & 184 & 359 & 1 \\
\hline 50 & 3156 & 9 & 79 & 12988 & 36 & 119 & 128 & 0 & 185 & 4544 & 13 \\
\hline 51 & 3025 & 8 & 80 & 1493 & 4 & 120 & 115 & 0 & 186 & 1893 & 5 \\
\hline 52 & 2005 & 6 & 81 & 1001 & 3 & 124 & 280 & 1 & 187 & 4572 & 13 \\
\hline 53 & 1052 & 3 & 82 & 154 & 0 & 131 & 77 & 0 & 188 & 1673 & 5 \\
\hline 54 & 206 & 1 & 88 & 115 & 0 & 140 & 77 & 0 & 211 & 1428 & 4 \\
\hline 60 & 154 & 0 & 89 & 257 & 1 & 142 & 77 & 0 & 212 & 309 & 1 \\
\hline 61 & 628 & 2 & 90 & 154 & 0 & 147 & 77 & 0 & 355 & 189 & 1 \\
\hline 62 & 1286 & 4 & 91 & 243 & 1 & & & & & & \\
\hline
\end{tabular}




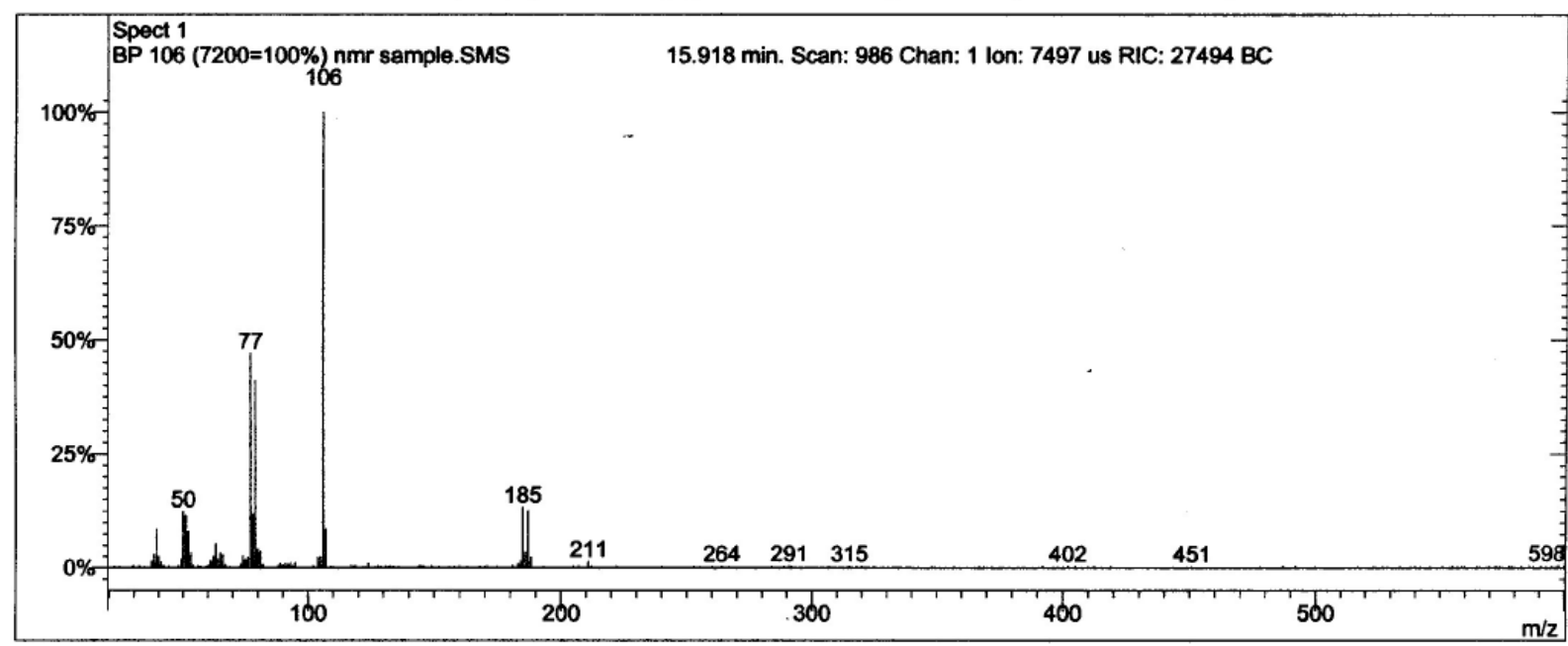

Spectrum from c:1... Ipyridine unsymml2,51nmr sample.sms Scan No: 986, Time: 15.918 minutes

No averaging. Background corrected.

Comment: 15.918 min. Scan: 986 Chan: 1 lon: 7497 us RIC: 27494 BC

Pair Count: 166 MW: 0 Formula: None CAS No: None Acquired Range: 20 - 600

\begin{tabular}{|c|c|c|c|c|c|c|c|c|c|c|c|}
\hline Ion & Int & \%BP & Ion & Int & \% BP & Ion & Int & $8 B P$ & Ion & Int & \% $\mathrm{BP}$ \\
\hline 22 & 23 & 0 & 88 & 36 & 1 & 181 & 36 & 1 & 487 & 23 & 0 \\
\hline 24 & 18 & 0 & 89 & 66 & 1 & 183 & 58 & 1 & 492 & 23 & 0 \\
\hline 29 & 22 & 0 & 90 & 43 & 1 & 184 & 110 & 2 & 500 & 14 & 0 \\
\hline 30 & 31 & 0 & 91 & 67 & 1 & 185 & 960 & 13 & 517 & 16 & 0 \\
\hline 32 & 28 & 0 & 92 & 51 & 1 & 186 & 250 & 3 & 523 & 20 & 0 \\
\hline 34 & 19 & 0 & 93 & 74 & 1 & 187 & 914 & 13 & 524 & 18 & 0 \\
\hline 37 & 105 & 1 & 94 & 30 & 0 & 188 & 170 & 2 & 527 & 16 & 0 \\
\hline 38 & 216 & 3 & 95 & 72 & 1 & 193 & 23 & 0 & 529 & 24 & 0 \\
\hline 39 & 619 & 9 & 102 & 33 & 0 & 205 & 23 & 0 & 534 & 26 & 0 \\
\hline 40 & 181 & 3 & 104 & 171 & 2 & 207 & 28 & 0 & 538 & 23 & 0 \\
\hline 41 & 84 & 1 & 105 & 174 & 2 & 210 & 14 & 0 & 540 & 23 & 0 \\
\hline 42 & 29 & 0 & 106 & 7200 & 100 & 211 & 98 & 1 & 542 & 16 & 0 \\
\hline 44 & 28 & 0 & 107 & 614 & 9 & 212 & 30 & 0 & 546 & 26 & 0 \\
\hline 48 & 33 & 0 & 109 & 21 & 0 & 222 & 23 & 0 & 551 & 21 & 0 \\
\hline 49 & 144 & 2 & 110 & 21 & 0 & 240 & 20 & 0 & 554 & 14 & 0 \\
\hline 50 & 892 & 12 & 117 & 34 & 0 & 253 & 19 & 0 & 555 & 24 & 0 \\
\hline 51 & 828 & 12 & 118 & 30 & 0 & 255 & 11 & 0 & 557 & 26 & 0 \\
\hline 52 & 583 & 8 & 119 & 33 & 0 & 260 & 20 & 0 & 559 & 26 & 0 \\
\hline 53 & 239 & 3 & 122 & 17 & 0 & 264 & 23 & 0 & 560 & 25 & 0 \\
\hline 54 & 43 & 1 & 123 & 20 & 0 & 267 & 16 & 0 & 565 & 23 & 0 \\
\hline 56 & 30 & 0 & 124 & 70 & 1 & 284 & 16 & 0 & 566 & 18 & 0 \\
\hline 57 & 25 & 0 & 127 & 18 & 0 & 289 & 16 & 0 & 569 & 20 & 0 \\
\hline 59 & 20 & 0 & 128 & 30 & 0 & 291 & 33 & 0 & 570 & 23 & 0 \\
\hline 60 & 36 & 1 & 129 & 23 & 0 & 292 & 26 & 0 & 572 & 24 & 0 \\
\hline 61 & 116 & 2 & 131 & 30 & 0 & 307 & 20 & 0 & 573 & 25 & 0 \\
\hline 62 & 183 & 3 & 132 & 22 & 0 & 315 & 23 & 0 & 574 & 21 & 0 \\
\hline 63 & 381 & 5 & 133 & 30 & 0 & 318 & 16 & 0 & 575 & 17 & 0 \\
\hline 64 & 136 & 2 & 134 & 24 & 0 & 320 & 20 & 0 & 578 & 28 & 0 \\
\hline 65 & 232 & 3 & 136 & 16 & 0 & 325 & 16 & 0 & 579 & 23 & 0 \\
\hline 66 & 203 & 3 & 144 & 33 & 0 & 348 & 16 & 0 & 580 & 17 & 0 \\
\hline 67 & 44 & 1 & 145 & 36 & 1 & 357 & 15 & 0 & 583 & 21 & 0 \\
\hline 69 & 20 & 0 & 146 & 23 & 0 & 367 & 16 & 0 & 586 & 33 & 0 \\
\hline 73 & 60 & 1 & 149 & 33 & 0 & 369 & 16 & 0 & 588 & 21 & 0 \\
\hline 74 & 193 & 3 & 152 & 18 & 0 & 392 & 20 & 0 & 590 & 13 & 0 \\
\hline 75 & 133 & 2 & 156 & 16 & 0 & 402 & 23 & 0 & 591 & 23 & 0 \\
\hline 76 & 173 & 2 & 158 & 20 & 0 & 405 & 21 & 0 & 593 & 19 & 0 \\
\hline 77 & 3384 & 47 & 160 & 33 & 0 & 410 & 15 & 0 & 595 & 23 & 0 \\
\hline 78 & 855 & 12 & 163 & 24 & 0 & 412 & 16 & 0 & 596 & 20 & 0 \\
\hline 79 & 2955 & 41 & 168 & 23 & 0 & 419 & 16 & 0 & 597 & 17 & 0 \\
\hline
\end{tabular}




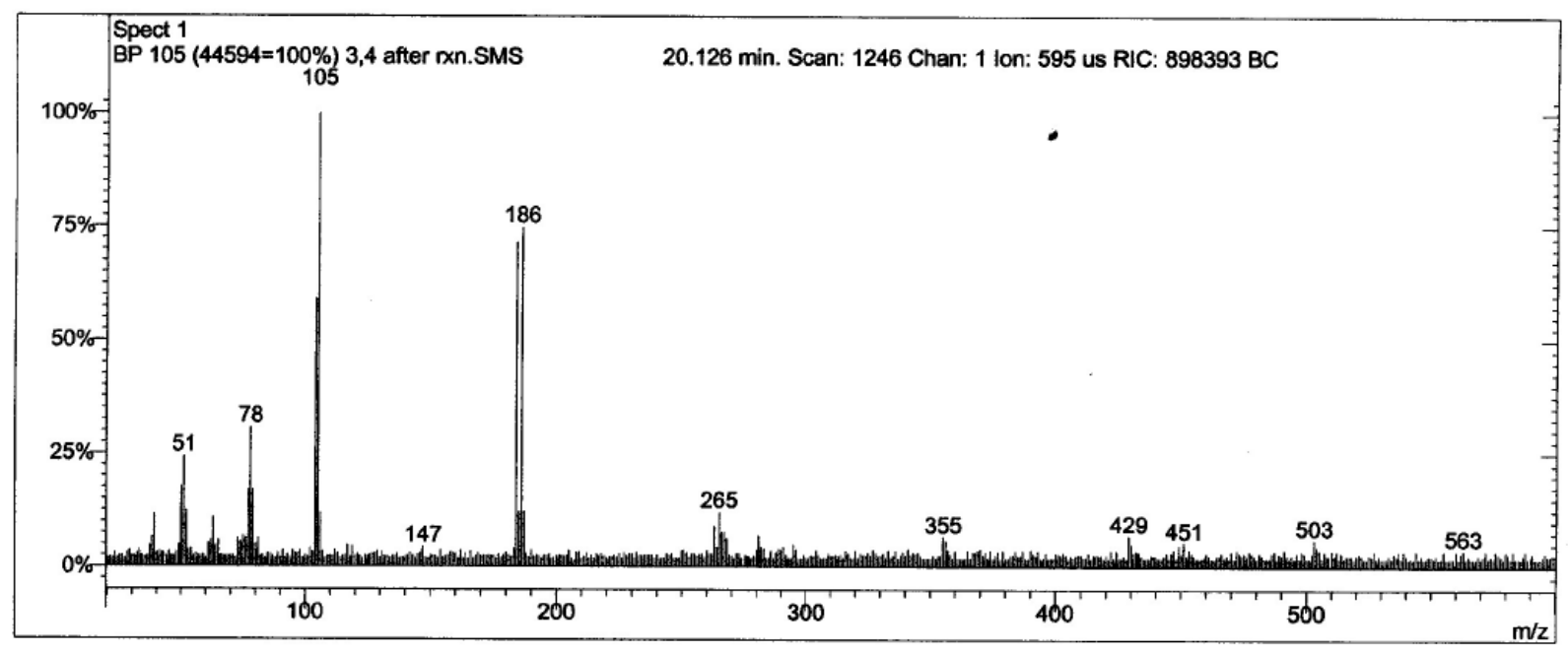

Spectrum from c:1... 13,4-lut bromination 13,4 after rxn.sms

Scan No: 1246, Time: 20.126 minutes

No averaging. Background corrected.

Comment: 20.126 min. Scan: 1246 Chan: 1 lon: 595 us RIC: 898393 BC

Pair Count: 581 MW: 0 Formula: None CAS No: None Acquired Range: 20 - 600

\begin{tabular}{|c|c|c|c|c|c|c|c|c|c|c|c|}
\hline Ion & Int & $\% \mathrm{BP}$ & Ion & Int & $\%$ BP & Ion & Int & $8 \mathrm{BP}$ & Ion & Int & $\frac{8}{8} \mathrm{BP}$ \\
\hline 20 & 819 & 2 & 166 & 1549 & 3 & 311 & 1066 & 2 & 456 & 834 & 2 \\
\hline 21 & 895 & 2 & 167 & 785 & 2 & 312 & 1081 & 2 & 457 & 819 & 2 \\
\hline 22 & 857 & 2 & 168 & 1055 & 2 & 313 & 1026 & 2 & 458 & 826 & 2 \\
\hline 23 & 1415 & 3 & 169 & 1365 & 3 & 314 & 1128 & 3 & 459 & 1038 & 2 \\
\hline 24 & 865 & 2 & 170 & 1059 & 2 & 315 & 838 & 2 & 460 & 1304 & 3 \\
\hline 25 & 1093 & 2 & 171 & 1082 & 2 & 316 & 1548 & 3 & 461 & 1103 & 2 \\
\hline $26^{\circ}$ & 1079 & 2 & 172 & 1068 & 2 & 317 & 1311 & 3 & 462 & 797 & 2 \\
\hline 27 & 825 & 2 & 173 & 1117 & 3 & 318 & 827 & 2 & 463 & 821 & 2 \\
\hline 28 & 1363 & 3 & 174 & 1048 & 2 & 319 & 837 & 2 & 464 & 1057 & 2 \\
\hline 29 & 1566 & 4 & 175 & 1065 & 2 & 320 & 1590 & 4 & 465 & 1073 & 2 \\
\hline 30 & 1122 & 3 & 176 & 820 & 2 & 321 & 838 & 2 & 466 & 1322 & 3 \\
\hline 31 & 1050 & 2 & 177 & 1270 & 3 & 322 & 1302 & 3 & 467 & 834 & 2 \\
\hline 32 & 1343 & 3 & 178 & 834 & 2 & 323 & 1144 & 3 & 468 & 1104 & 2 \\
\hline 33 & 1665 & 4 & 179 & 1134 & 3 & 324 & 1104 & 2 & 469 & 828 & 2 \\
\hline 34 & 1135 & 3 & 180 & 1394 & 3 & 325 & 1421 & 3 & 470 & 1544 & 3 \\
\hline 35 & 895 & 2 & 181 & 1119 & 3 & 326 & 1069 & 2 & 471 & 811 & 2 \\
\hline 36 & 1071 & 2 & 182 & 948 & 2 & 327 & 1682 & 4 & 472 & 1644 & 4 \\
\hline 37 & 2029 & 5 & 183 & 1837 & 4 & 328 & 1329 & 3 & 473 & 1348 & 3 \\
\hline 38 & 2865 & 6 & 184 & 31890 & 72 & 329 & 1026 & 2 & 474 & 1122 & 3 \\
\hline 39 & 5170 & 12 & 185 & 5443 & 12 & 330 & 1101 & 2 & 475 & 1329 & 3 \\
\hline 40 & 1355 & 3 & 186 & 33331 & 75 & 331 & 1314 & 3 & 476 & 1037 & 2 \\
\hline 41 & 1464 & 3 & 187 & 5437 & 12 & 332 & 841 & 2 & 477 & 1557 & 3 \\
\hline 42 & 1372 & 3 & 188 & 1353 & 3 & 333 & 1608 & 4 & 478 & 1318 & 3 \\
\hline 43 & 1416 & 3 & 189 & 925 & 2 & 334 & 846 & 2 & 479 & 1078 & 2 \\
\hline 44 & 1020 & 2 & 190 & 1582 & 4 & 335 & 1042 & 2 & 480 & 796 & 2 \\
\hline 45 & 1482 & 3 & 191 & 1003 & 2 & 336 & 1593 & 4 & 481 & 1321 & 3 \\
\hline 46 & 1059 & 2 & 192 & 1121 & 3 & 337 & 822 & 2 & 482 & 1142 & 3 \\
\hline 47 & 1024 & 2 & 193 & 1056 & 2 & 338 & 1143 & 3 & 483 & 792 & 2 \\
\hline 48 & 1458 & 3 & 194 & 808 & 2 & 339 & 1475 & 3 & 484 & 845 & 2 \\
\hline 49 & 2134 & 5 & 195 & 1093 & 2 & 340 & 1070 & 2 & 485 & 849 & 2 \\
\hline 50 & 7884 & 18 & 196 & 1085 & 2 & 341 & 1742 & 4 & 486 & 1330 & 3 \\
\hline 51 & 10802 & 24 & 197 & 1290 & 3 & 342 & 1111 & 2 & 487 & 1571 & 4 \\
\hline 52 & 5490 & 12 & 198 & 827 & 2 & 343 & 1391 & 3 & 488 & 776 & 2 \\
\hline 53 & 1642 & 4 & 199 & 839 & 2 & 344 & 1196 & 3 & 489 & 1274 & 3 \\
\hline 54 & 1757 & 4 & 200 & 1052 & 2 & 345 & 1423 & 3 & 490 & 1083 & 2 \\
\hline 55 & 1059 & 2 & 201 & 1124 & 3 & 346 & 1018 & 2 & 491 & 1702 & 4 \\
\hline 56 & 1286 & 3 & 202 & 1084 & 2 & 347 & 817 & 2 & 492 & 1073 & 2 \\
\hline 57 & 1083 & 2 & 203 & 1047 & 2 & 348 & 824 & 2 & 493 & 821 & 2 \\
\hline 58 & 1073 & 2 & 204 & 1066 & 2 & 349 & 820 & 2 & 494 & 1099 & 2 \\
\hline
\end{tabular}




\section{References}

(1) Waki, Y.; Tanaka, H. J. Org. Chem. 2003, 68, 3938-3942.

(2) Tao, X.; Qian, Y.; Huang, J. Chemical Communications 2003, 1444-1445.

(3) Walker, J. Chem. Rev. 1993, 93, 1937-1944.

(4) Gribble, G. Chem. Soc. Rev. 1999, 28, 335-346.

(5) Carey, F.; sundberg, R. Advanced Organic Chemistry; 1990; Vol. third edition, pp 691.

(6) Traynham, J. G.; Lee, Y. J. Am. Chem. Soc. 1974, 96, 3590-3594.

(7) Dauben, H. J.; McCoy, L. L. J. Am. Chem. Soc. 1959, 81, 4863-4873.

(8) Pizey J.S. 1974, PP 1-63.

(9) Djerassi, C. Chem. Rev. 1943, 43, 271-317.

(10) Kharasch, M. S.; Brown, H. C. J. Am. Chem. Soc. 1940, 62, 925-929.

(11) Howton, D. R. J. Am. Chem. Soc. 1947, 69, 2060-2061.

(12) Day, J. C.; Lindstrom, M. J.; Skell, P. S. J. Am. Chem. Soc. 1974, 96, 5616-5617.

(13) Xu, B.; Zhao, S. Synthesis-Stuttgart 2000, 139-143.

(14) Frank-Neumann M Tetrahedron Lett. 1989, 30, 6679.

(15) Martin, R.; chapleo, C.; Svanholt, K.; Dreiding, A. Helv. Chim. Acta 1976, 59, 2724-2727.

(16) Yoda H, Shirakawa K, Takabe K CL 1989, 1391.

(17) Hahn, R. C.; Corbin, T. F.; Shechter, H. J. Am. Chem. Soc. 1968, 90, 3404-3415.

(18) Leed, A. R.; Boettger, S. D.; Ganem, B. J. Org. Chem. 1980, 45, 1098-1106.

(19) Gribble, G. W.; Keavy, D. J. et. al. J. J. Org. Chem. 1992, 57, 5878-5891.

(20) Scrivanti, A. Journal of the Chemical Society-Perkin Transactions 1 1996, 307-311.

(21) Araki, K.; Matsumoto, H.; Harada, T.; Shinkai, S. J. Org. Chem. 1995, 60, 4862-4867.

(22) Harth, H. J. Org. Chem. 1991, 56, 5630-5640. 
(23) Timko, J.; Walba, D.; Cram, D. J. Am. Chem. Soc. 1977, 99, 6392-6398.

(24) Barnes, R. A.; Fales, H. M. J. Am. Chem. Soc. 1953, 75, 3830-3831.

(25) Offermann, W.; Vogtle, F. Synthesis-Stuttgart 1977, 272-273.

(26) Liu, P.; Chen, Y.; Deng, J.; Tu, Y. Synthesis 2001, 2001, 2078-2080.

(27) Weingarten, M. et.al. Angewandte Chemie-International Edition 1998, 37, 1846-1850.

(28) Offermann, W.; Voegtle, F. J. Org. Chem. 1979, 44, 710-713.

(29) Zuber, M.; Lichent, RG Chemisch Berichte-Recueil 1973, 106, 717-718.

(30) Filler, R. Chem. Rev. 1963, 63, 21-43.

(31) Jones, T.; Fales, H. Tetrahedron Lett. 1983, 24, 5439-5440.

(32) Gruter, G. M.; Akkerman, O. S.; Bickelhaupt, F. J. Org. Chem. 1994, 59, 4473-4481.

(33) Jung, F.; Ladjama, D.; Riehl, J. Synthesis-sStuttgart 1979, 507-508.

(34) Viehe, H. et. al. Angewandte Chemie-International Edition in Engligh 1979, 18, 917-932.

(35) Suzuki, T. et.al. Bull. Chem. Soc. Jpn. 1985, 58, 2812-2820.

(36) Gronowitz, S. Synthesis-Stuttgart 1980, 322-324.

(37) Bretschneider, T.; Miltz, W.; Munster, R.; Steglich, W. Tetrahedron 1988, 44, 5403-5414.

(38) Bao, L.; Coyle, C.; Gleason, J.; Horovitch, S. Organic Synthesis 1988, 50-9, 190-193.

(39) Bao, L.; BLack, C.; Gleason, J.; Smith, R. J. Org. Chem. 1975, 40, 3420-3427.

(40) HILL, K. J. Org. Chem. 1973, 38, 2576-2578.

(41) Amice, P.; Conia, J. Synthesis-Sttugart 1976, 194-196.

(42) Bridson, J. Canadian Journal of Chemistry 1972, 50, 2387.

(43) Ellman, J.; DOROW, R. Tetrahedron Lett. 1987, 28, 1123-1126.

(44) Dudfield, P. Tetrahedron Lett. 1985, 26, 5037-5040.

(45) Murthy, K. Tetrahedron Lett. 1987, 28, 683-684. 
(46) Levy, A.; Talley, P.; Dunford, J. Tetrahedron Lett. 1977, 3545-3548.

(47) Zweifel, G.; On, H. Synthesis-Stuttgart 1980, 803-805.

(48) Camps, F.; Chamorro, E. et.al. Synthetic Communications 1989, 19, 3211-3220.

(49) Parsons, P.; Naylor, A. Synlett 1993, 189-190.

(50) Luh, T. J. Org. Chem. 1992, 57, 2760-2762.

(51) Annen, K. et.al. Angewandte Chemie-International Edition in English 1984, 23, 727-729.

(52) Mitchell, R. H.; Lai, Y.; Williams, R. V. J. Org. Chem. 1979, 44, 4733-4735.

(53) Ando, W.; Tsumaki, H. Synthesis-Stuttgart 1982, 263-264.

(54) Goldberg, Y.; Bensimon, C.; alper, H. J. Org. Chem. 1992, 57, 6374-6376.

(55) Ma, H.; Liu, Z.; Chen, S. Chinese Chemical Letters 2003, 14, 371-374.

(56) Carreno, M. C. et.al. J. Org. Chem. 1995, 60, 5328-5331.

(57) Dauben, H.; McCoy, L. J. Org. Chem. 1959, 24, 1577-1579.

(58) G. F. Bloomfield J. Chem. Soc. 1944, 14.

(59) Pearson, R. E.; Martin, J. C. J. Am. Chem. Soc. 1963, 85, 3142-3146.

(60) Fisher, T. H.; Meierhoefer, A. W. J. Org. Chem. 1978, 43, 220-224.

(61) Russell, G. A.; DeBoer, C.; Desmond, K. M. J. Am. Chem. Soc. 1963, 85, 365-366.

(62) Zupan, M.; Stavber, S. Helv. Chim. Acta 2009, 92, 555-566.

(63) Adam, J.; Gosselain, P. A.; Goldfinger, P. Nature 1953, 171, 704-705.

(64) Walling, C.; Rieger, A. L.; Tanner, D. D. J. Am. Chem. Soc. 1963, 85, 3129-3134.

(65) Vogtle, F. Synthesis-Stuttgart 1977, 272-273.

(66) Vogtle, F. Angewandte Chemie-International Edition in English 1980, 19, 464-465.

(67) Giese, B. Angewandte Chemie-International Edition in English 1977, 16, 125-136.

(68) Lustyk, J.; Ingold, K. J. Am. Chem. Soc. 1989, 111, 3652-3658. 
(69) Lusztyk, J.; Raner, K. Acc. Chem. Res. 1990, 23, 219-225.

(70) Perlmutter, H.; Gu, C.; Arco, S.; Quibuyen, T. J. Org. Chem. 1997, 62, 236-237.

(71) Paquette, L. A. Encyclopedia of reagents for organic synthesis; 1995 .

(72) van Klink, G.; van Koten, G. Green Chem. 2003, 5, 470-474.

(73) Ostrowski, P. et. al. Modern Solvents in Organic Synthesis 1999, 206, 79-105.

(74) Laval, G.; Tu, S.; Jiang, D.; Robinson, C.; Scott, R. Synthesis-Stuttgart 2009, 1807-1810.

(75) Zupan, M. J. Org. Chem. 1986, 51, 929-931.

(76) Kasliwal, R.; Kulkarni, V.; Neamati, N. Bioorganic 2004, 12, 2317-2333.

(77) Hirai, T. Synlett 2003, 702-704.

(78) Prashad, M. et.al. Synthetic Communications 1997, 27, 2159-2163.

(79) Natu, A.; Argade, N. J. Org. Chem. 1998, 63, 9557-9558.

(80) Kutney J. P.; Cretney, W.; Tabata, T.; Frank, M. Can. J. Chem. 1964, 42, 698.

(81) Basha, F.; Hashimoto, M.; Frye, R.; Kojo, S.; Hecht, S. J. Org. Chem. 1981, 46, 1413-1423.

(82) Adams, R.; Looker, J. H. J. Am. Chem. Soc. 1951, 73, 1145-1149.

(83) Deadly, L.; Reiss, J. J. Heterocycl. Chem. 1981, 18, 599-601.

(84) Ghera, E.; Ben David, Y.; Rapoport, H. J. Org. Chem. 1981, 46, 2059-2065.

(85) Strekowski, L.; Wydra, R. L.; Janda, L.; Harden, D. B. J. Org. Chem. 1991, 56, 5610-5614.

(86) Harden, D.; Grubb, W. et.al. J. Heterocycl. Chem. 1990, 27, 1393-1400.

(87) Hara, H.; Hirota, T.; Ohmori, S.; Yamamato, M. Chemical, 1975, 23, 2015-2018.

(88) Shadbolt, R. S. et.al. Journal of the Chemical Society C: Organic 1968, 1968, 733-740.

(89) Tesch, U. J. Heterocycl. Chem. 1977, 14, 1197-1198.

(90) Kharasch, M. S.; Zimmt, W. S.; Nudenberg, W. J. Org. Chem. 1955, 20, 1430-1442.

(91) Walling, C.; Jacknow, B. B. J. Am. Chem. Soc. 1960, 82, 6113-6115. 
(92) Thaler, W. J. Am. Chem. Soc. 1963, 85, 2607-2613.

(93) Kharasch, M. S.; SALLO, J. S.; Nudenberg, W. J. Org. Chem. 1956, 21, 129-135.

(94) Goering, H. L.; Sims, L. L. J. Am. Chem. Soc. 1955, 77, 3465-3469.

(95) Jaffé, H. H.; Doak, G. O. J. Am. Chem. Soc. 1955, 77, 4441-4444.

(96) Brown, H. C.; Okamoto, Y. J. Am. Chem. Soc. 1958, 80, 4979-4987.

(97) Russell, G. A.; Ito, A.; Hendry, D. G. J. Am. Chem. Soc. 1963, 85, 2976-2983.

(98) Maraschin, N. J. et.al. J. Am. Chem. Soc. 1975, 97, 513-517.

(99) Persico, D. F.; Lagow, R. J.; Clark, L. C.; Huang, H. J. Org. Chem. 1985, 50, 5156-5159.

(100) Newkome, G. Synthesis 1984, 1984, 676-679.

(101) Ghiaci M. and Asghari J. Bull. Chem. Soc. Jpn. 2001, 74, 1151-1152.

(102) Calandri, E. et.al. Anales De La Asociacion Quimica Argentina 1981, 69, 209-217.

(103) Klemm, L.; White, D.; Johnson, W. J. Heterocycl. Chem. 1972, 9, 843.

(104) Goswami, S.; Dey, S.; Jana, S.; Adak, A. Chem. Lett. 2004, 33, 916-917.

(105) Ijuin, R.; Umezawa, N.; Higuchi, T. Bioorg. Med. Chem. 2006, 14, 3563-3570.

(106) Murphy, J. M.; Liao, X.; Hartwig, J. F. J. Am. Chem. Soc. 2007, 129, 15434-15435.

(107) Pirondini Proceedings of the National Academy of Sciences 2002, 99, 4911.

(108) Shepherd, M. K. Journal of the Chemical Society, Perkin Transactions 1 1986, 1986, 1495-1498. 